\title{
SOCIAL ANALYSIS FOR POLITICAL PARTICIPATION OF RURAL YOUTH: A FIELD STUDY ON RURAL YOUTH SAMPLE IN ONE VILLAGE AT DAKAHLIA GOVERNORATE Abou- Hussein, lbtehal M.K. \\ Agricultural Extension and Rural Sociology Department, Faculty of Agriculture Mansoura University .
}

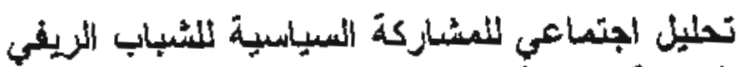

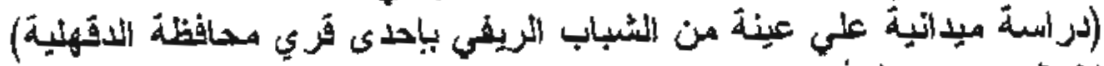

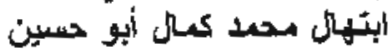

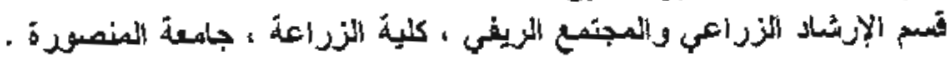

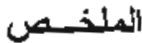

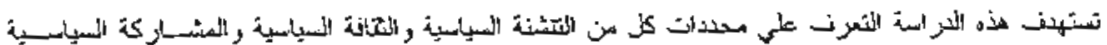

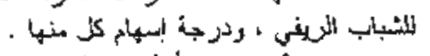

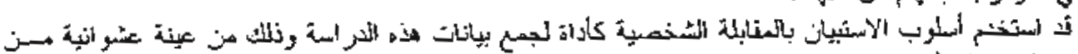

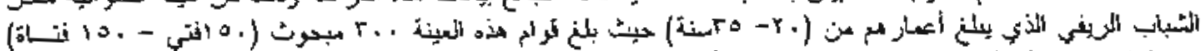

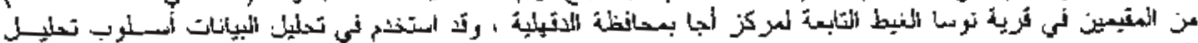

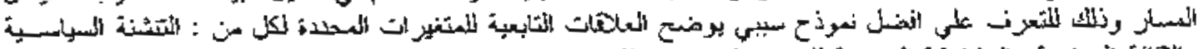

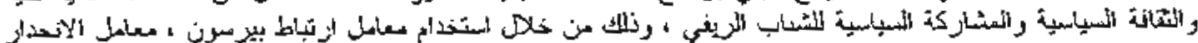

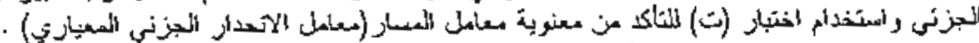

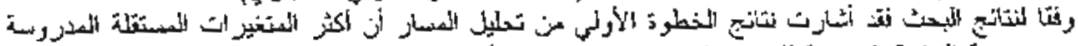

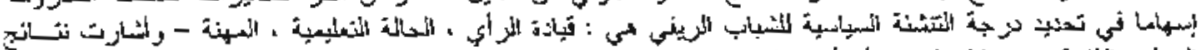

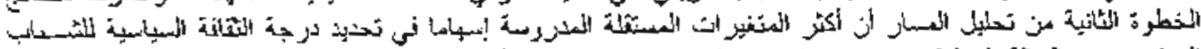

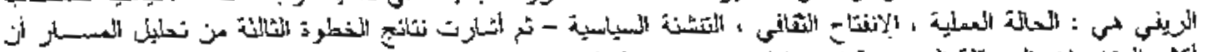

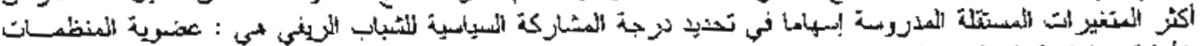

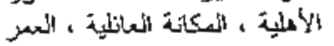

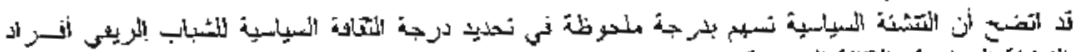

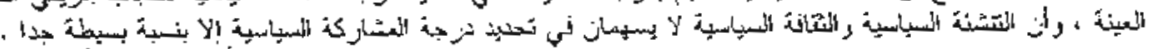

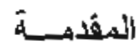

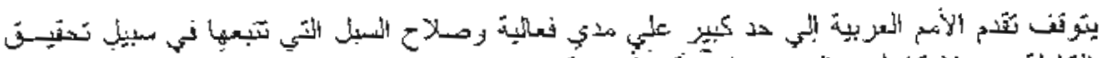

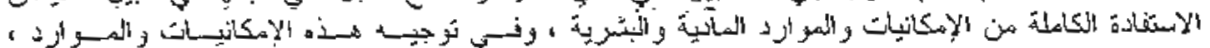

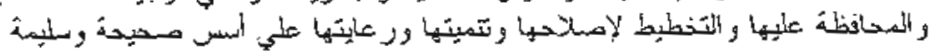

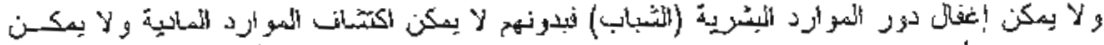

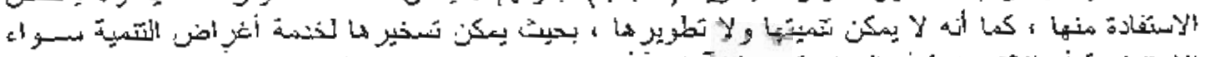

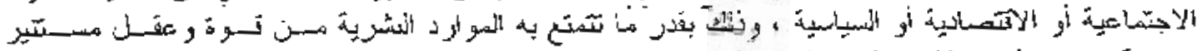

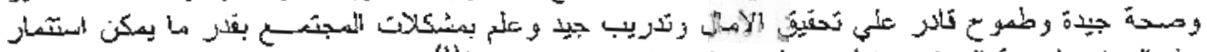

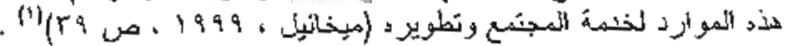

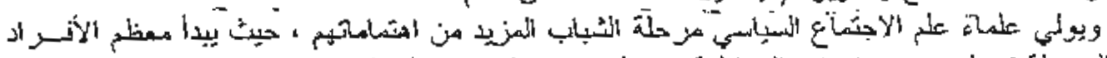

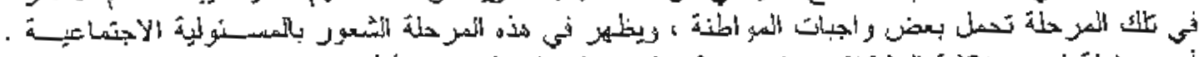

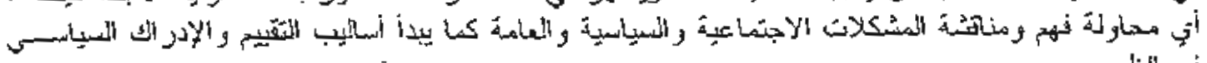

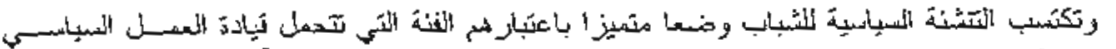

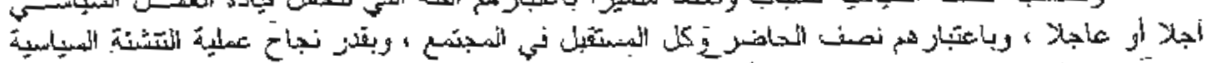

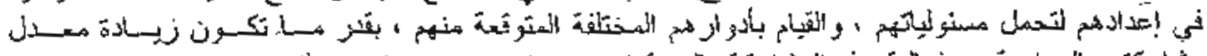

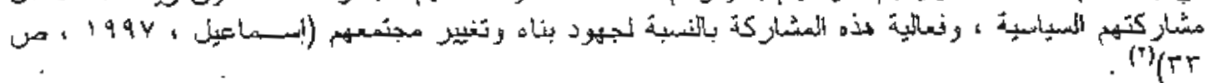




\section{Abou- Hussein, Ibtehal M.K.}

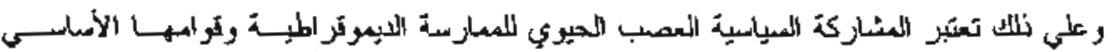

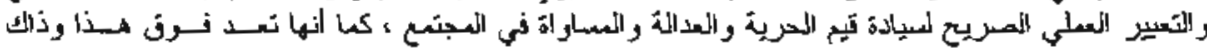

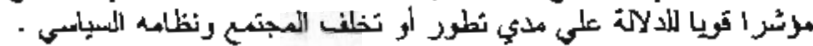

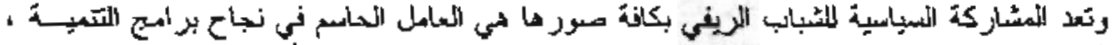

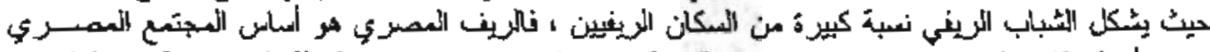

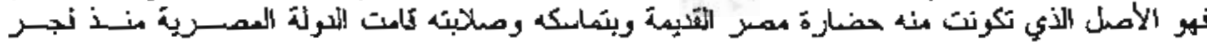

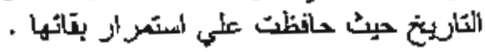

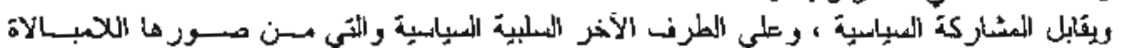

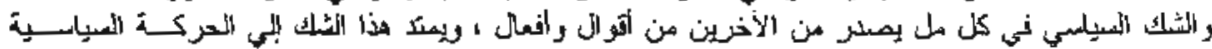

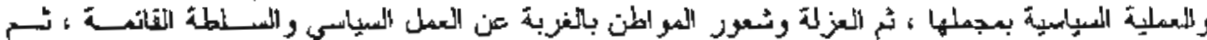

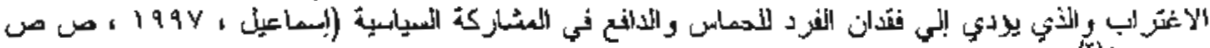

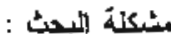

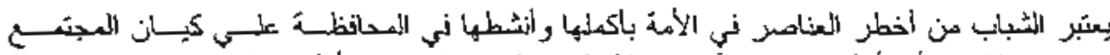

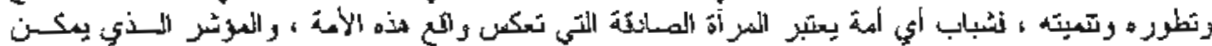

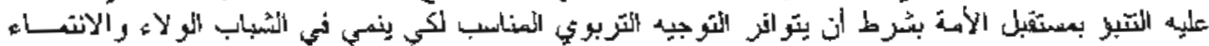

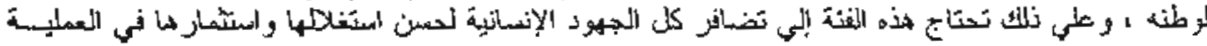

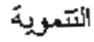

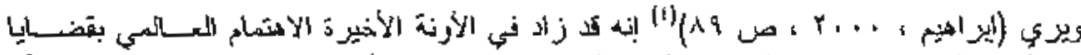

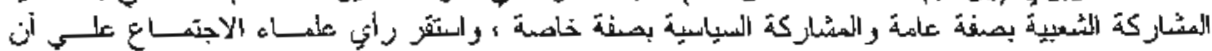

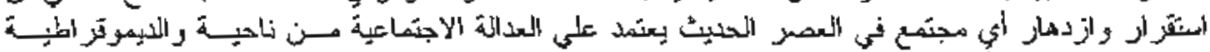

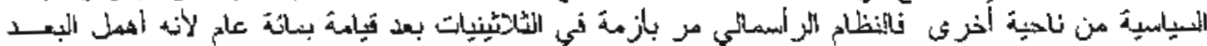

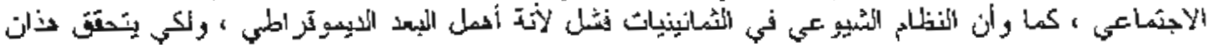

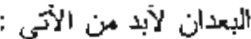

$$
\begin{aligned}
& \text { Y- حضور الجمالسيز ونعالبيتها. }
\end{aligned}
$$

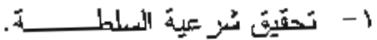

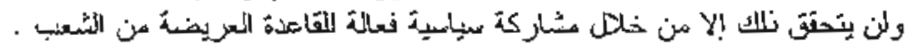

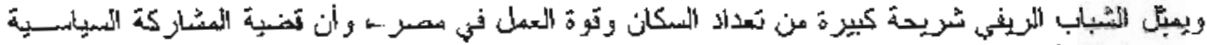

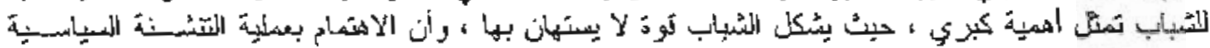

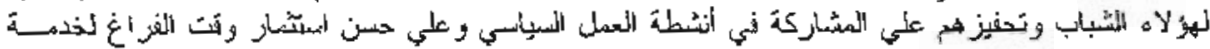

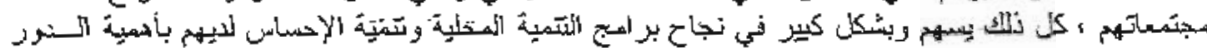

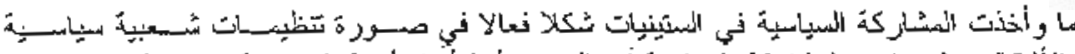

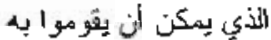

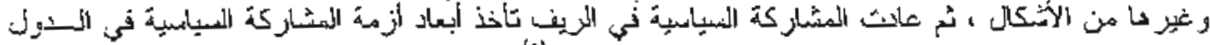

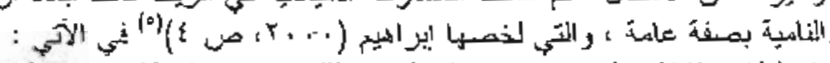

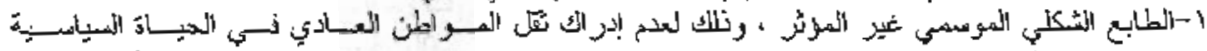

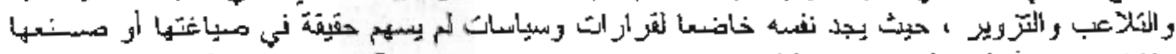

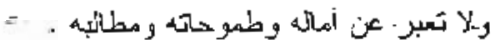

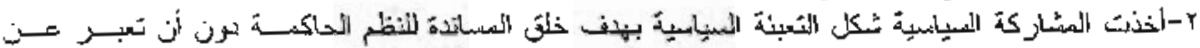

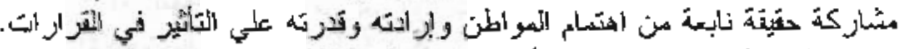

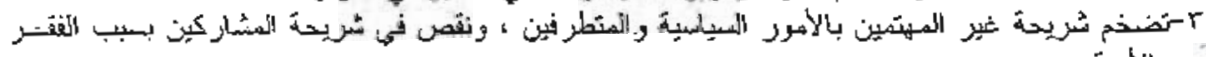

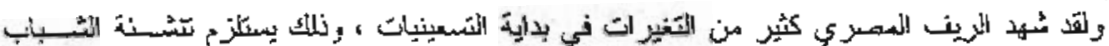

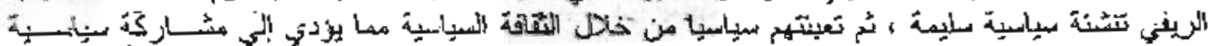

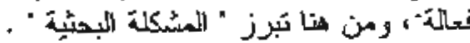




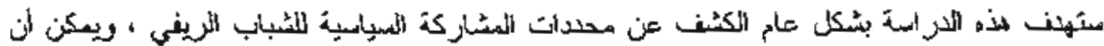

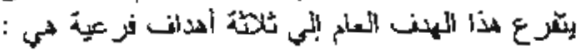

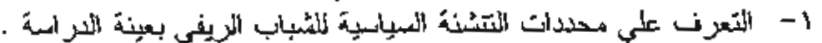

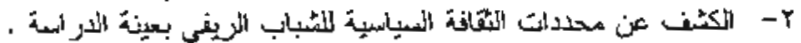

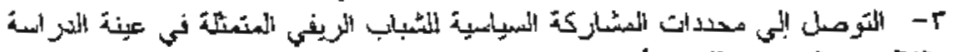

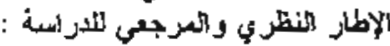

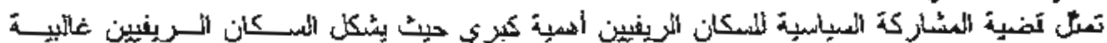

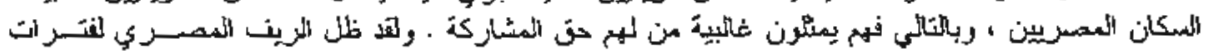

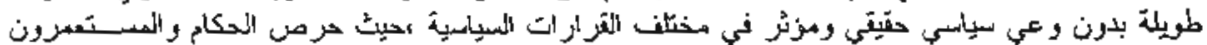

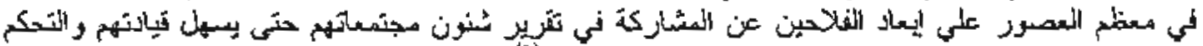

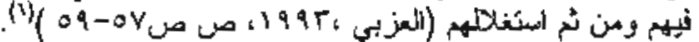

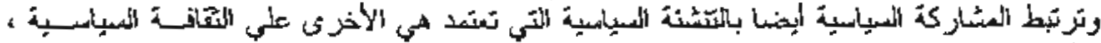

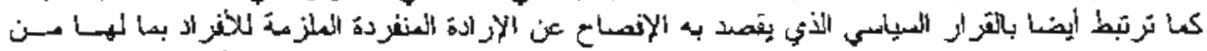

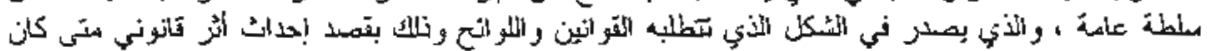

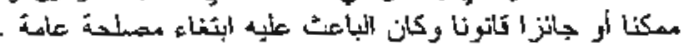

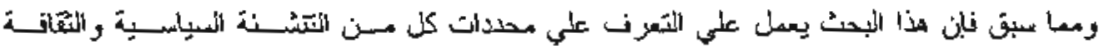

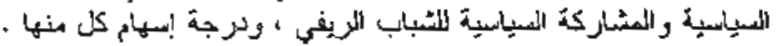

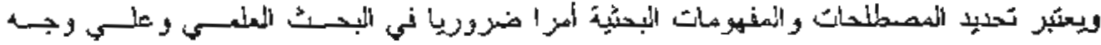

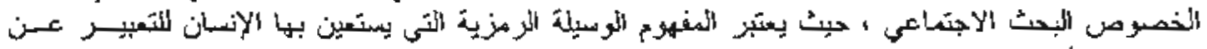

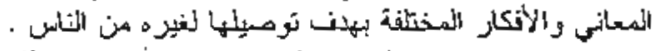

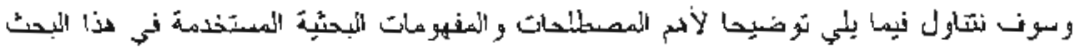

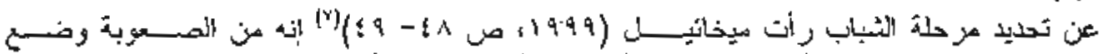

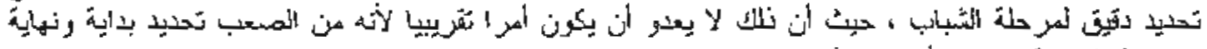

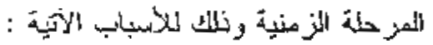

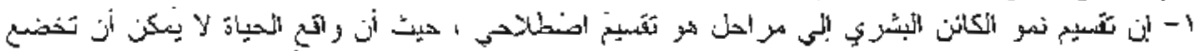

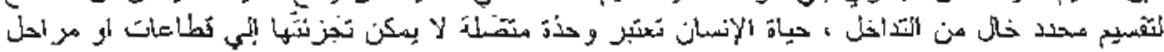

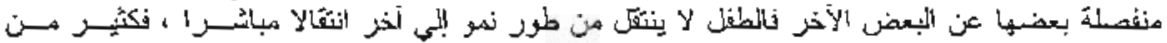

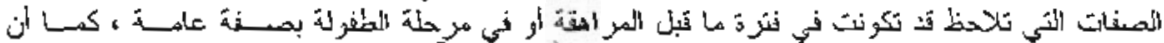

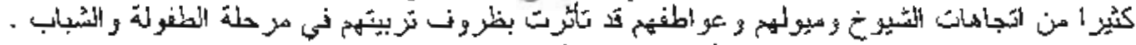

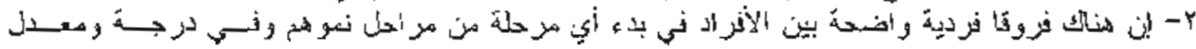

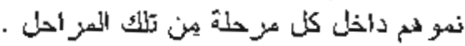

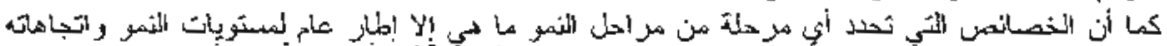

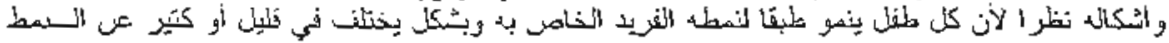

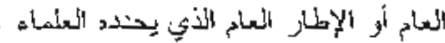

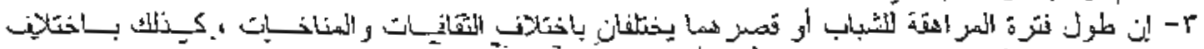

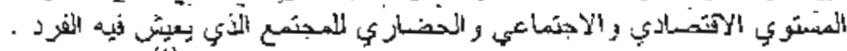

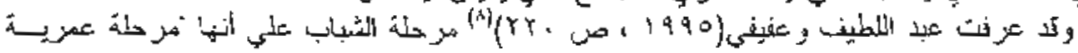

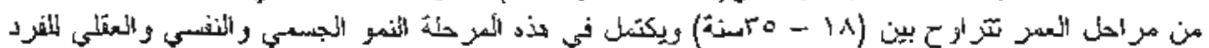

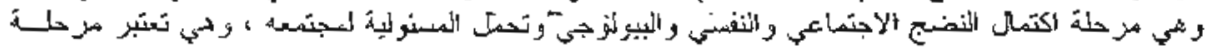

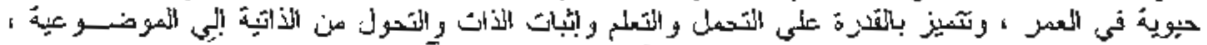

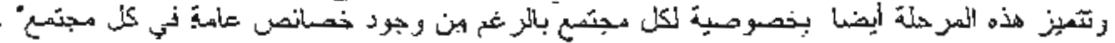

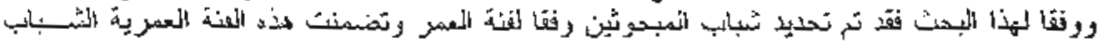

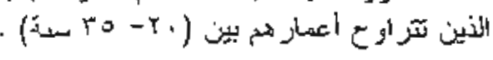


: أتخشئلة الفبيلسبة

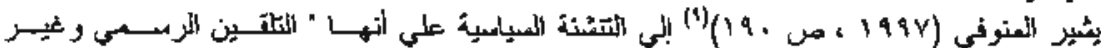

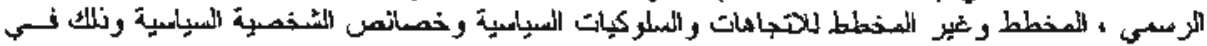

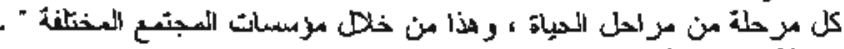

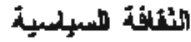

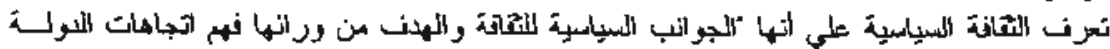

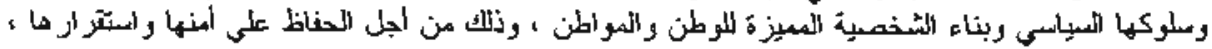

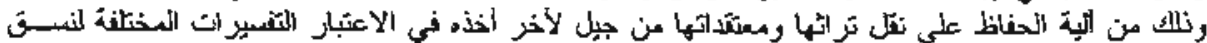

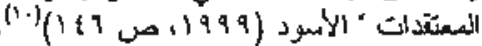

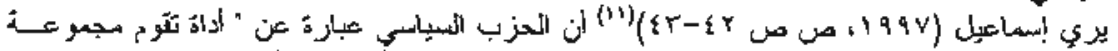

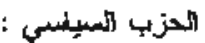

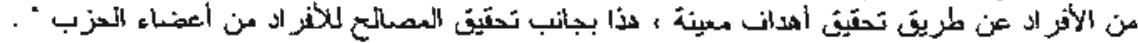

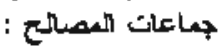

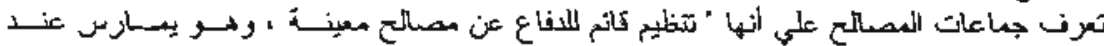

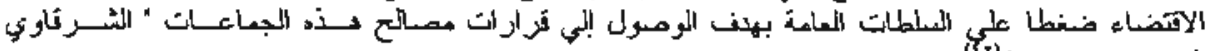

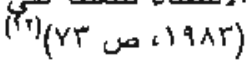

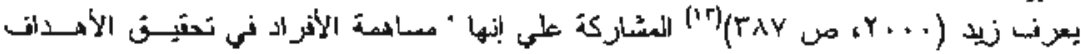
الهشياركة المثنعيبة :

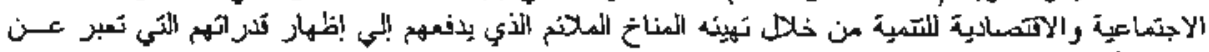

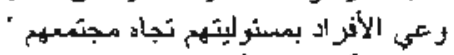

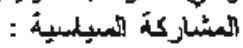

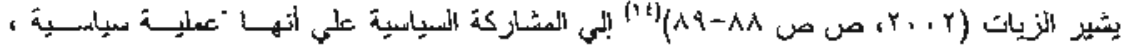

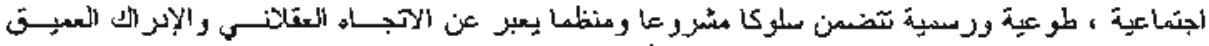

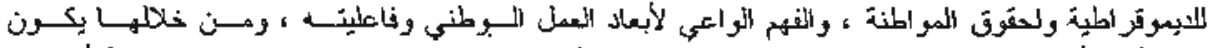

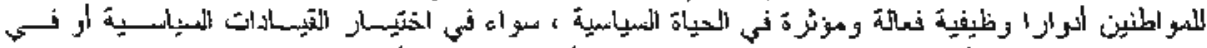

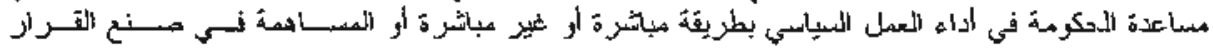

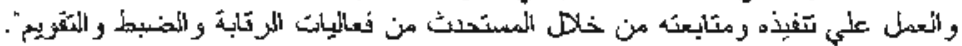

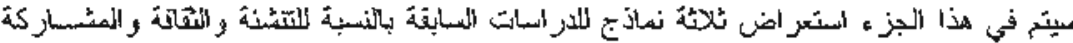

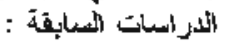

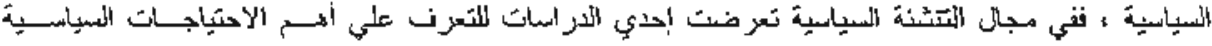

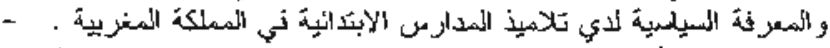

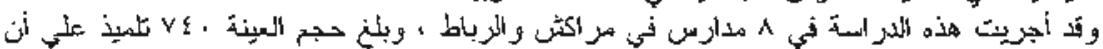

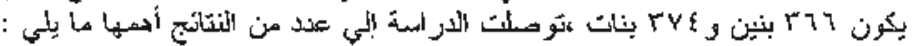

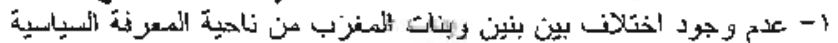

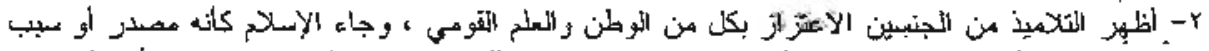

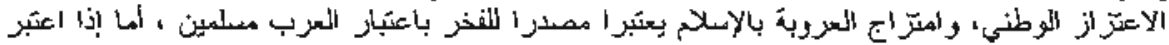

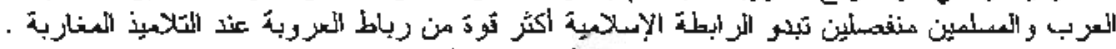

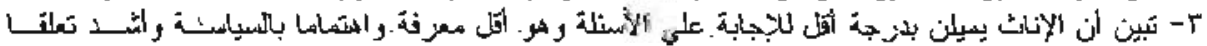
بالموطن

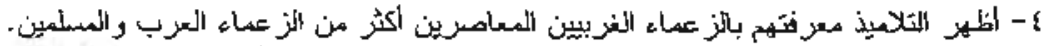

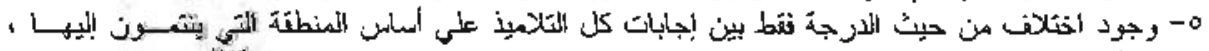

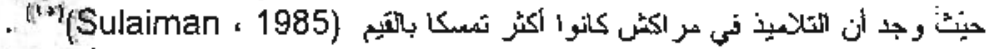

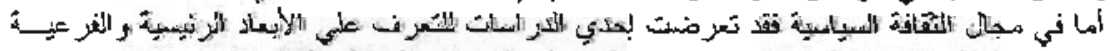

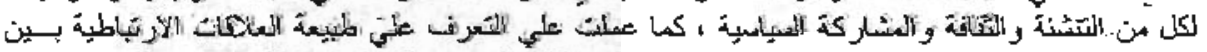

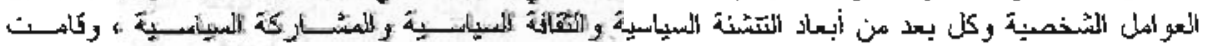

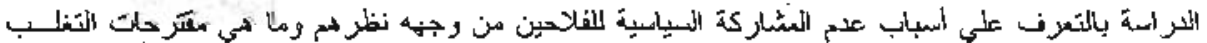

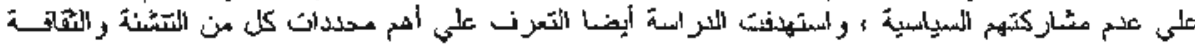




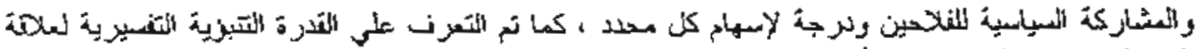

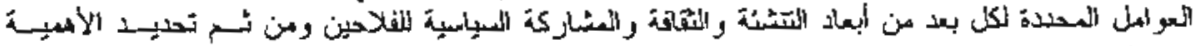

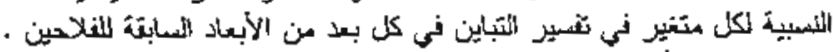

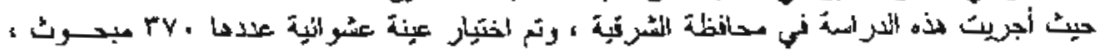

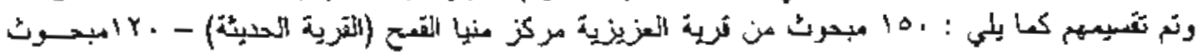

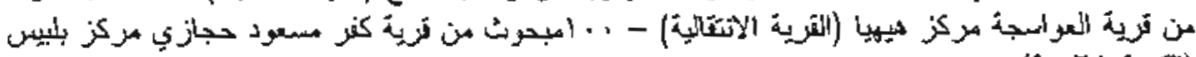

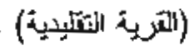

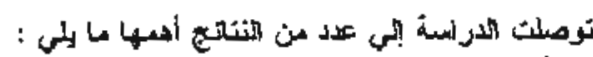

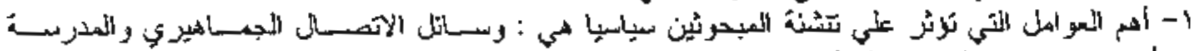

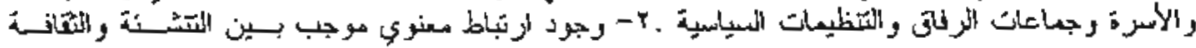

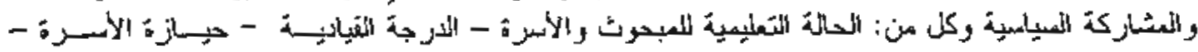

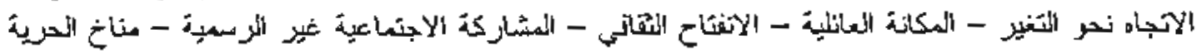

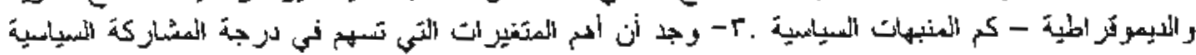

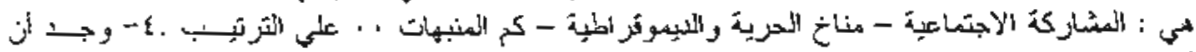

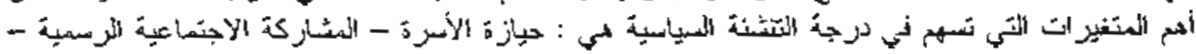

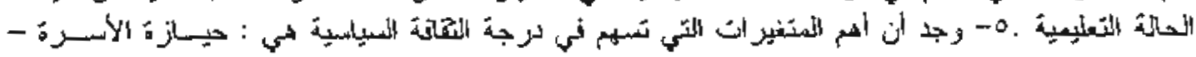

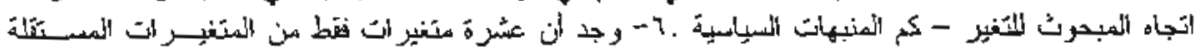

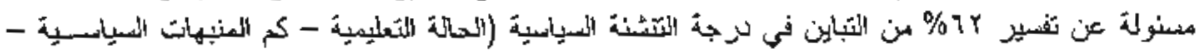

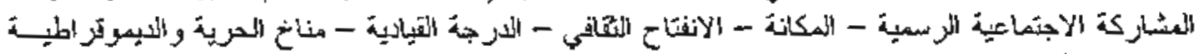

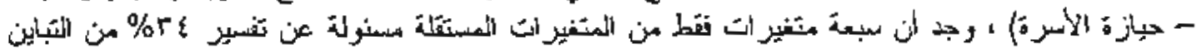

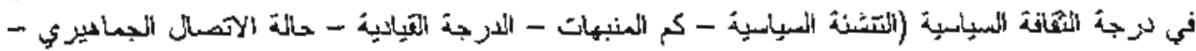

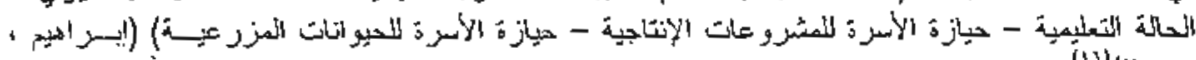
("ir)/ ( T....

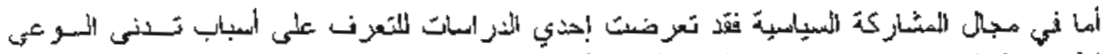

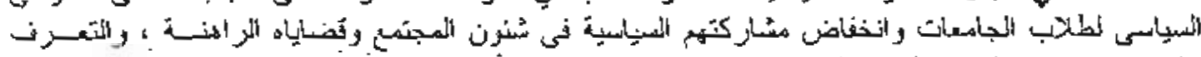

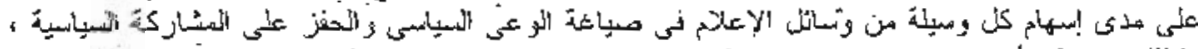

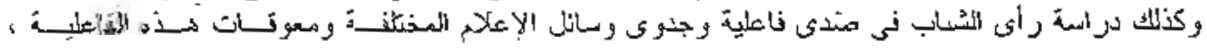

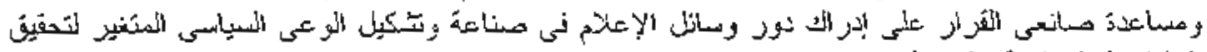
\% r व

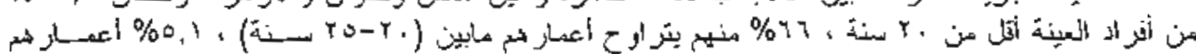

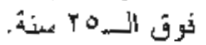

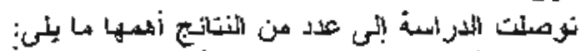

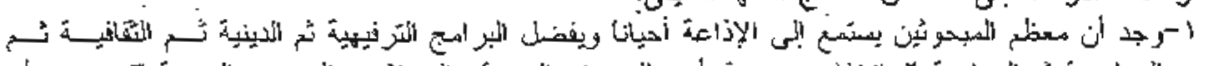

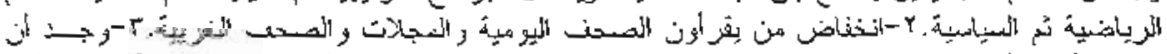

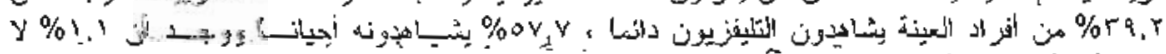

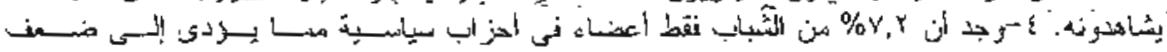

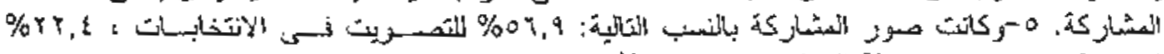

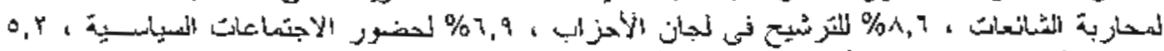

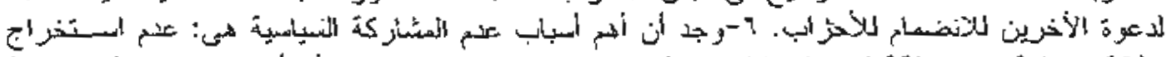

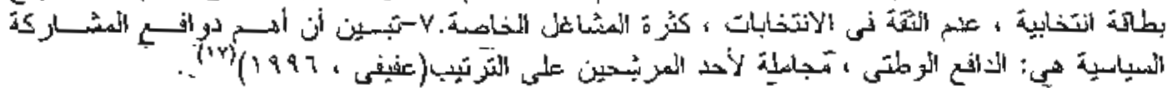

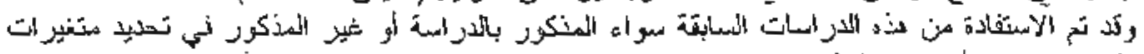

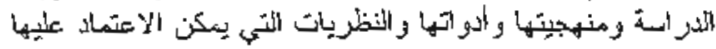


Abou- Hussein, Ibtehal M.K.

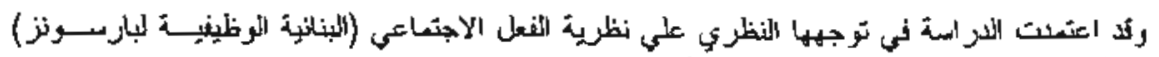

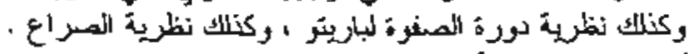

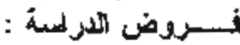

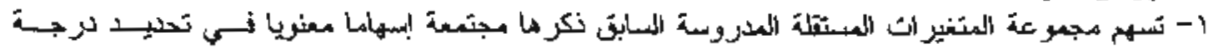

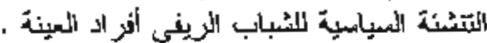

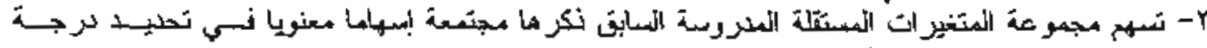

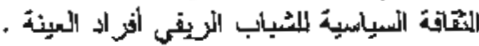

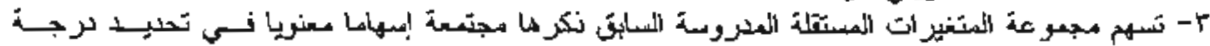

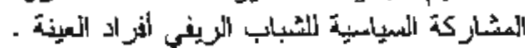

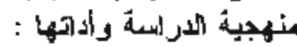

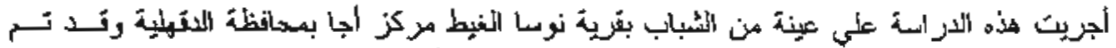

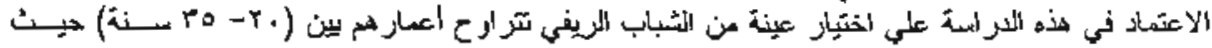

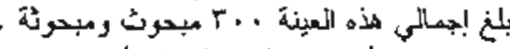

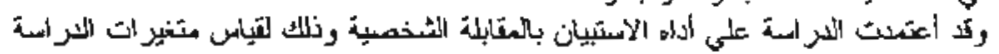

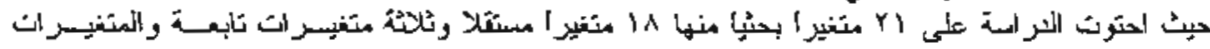

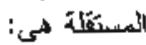

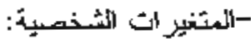
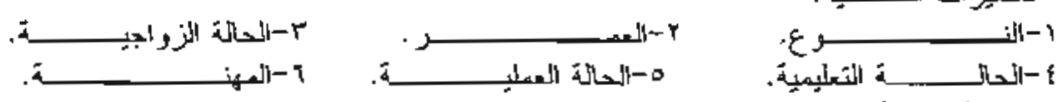

.

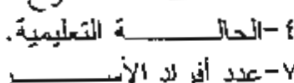

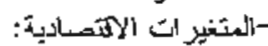

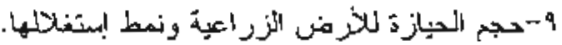

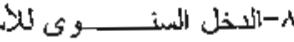

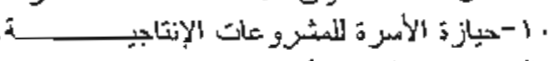

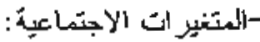

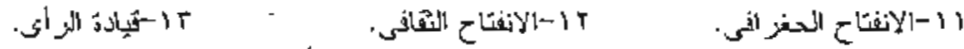

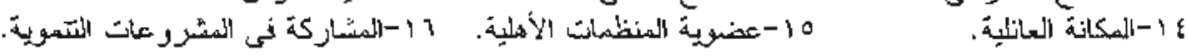

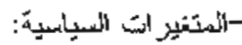

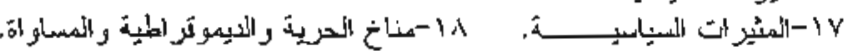

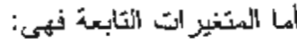

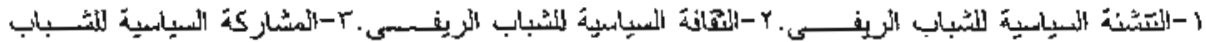

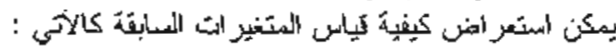

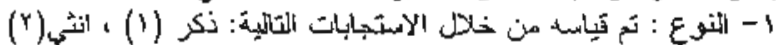

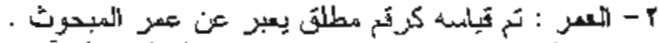

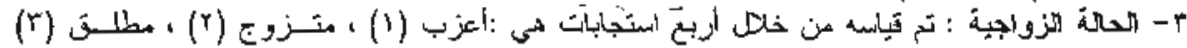

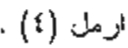

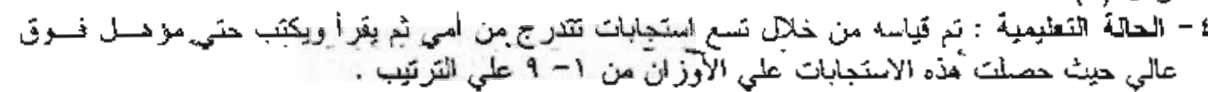

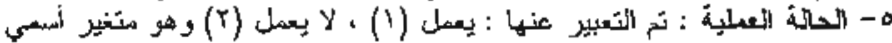

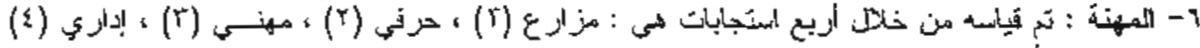

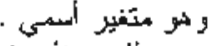

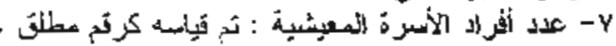

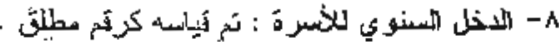

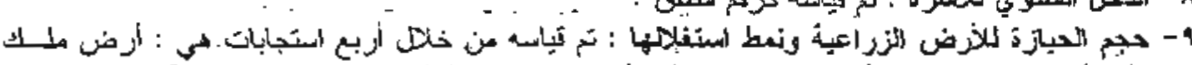

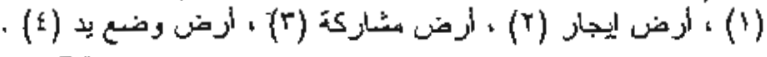




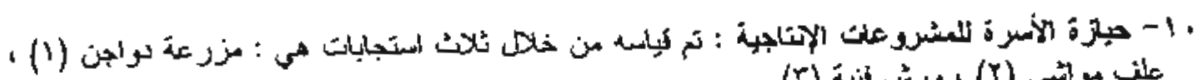

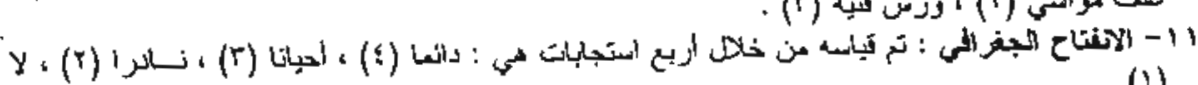

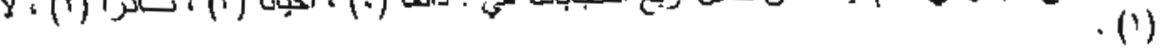

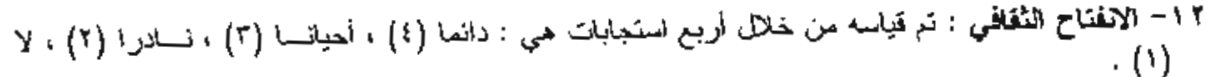

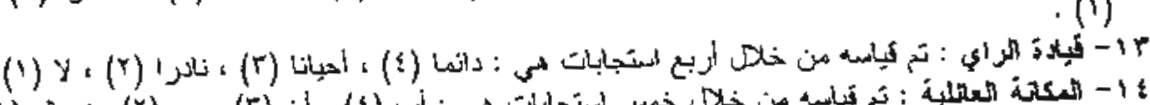

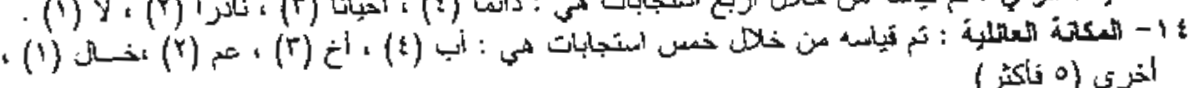

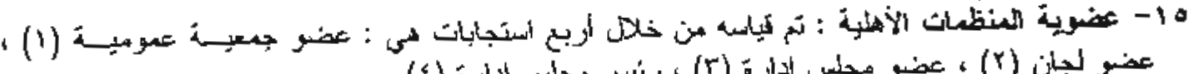

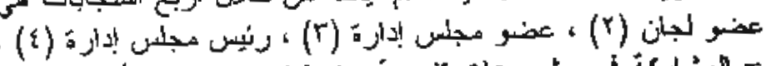

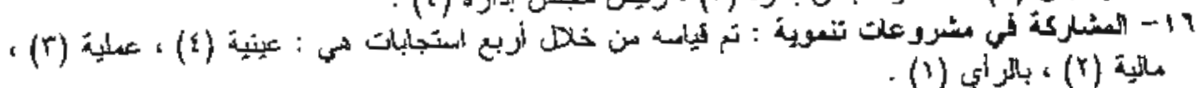

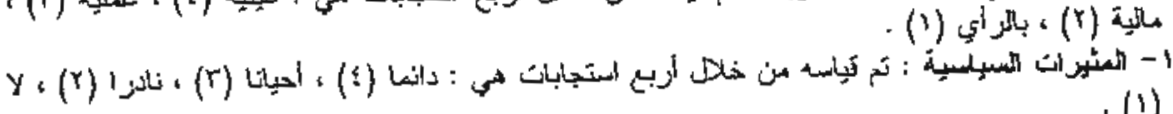

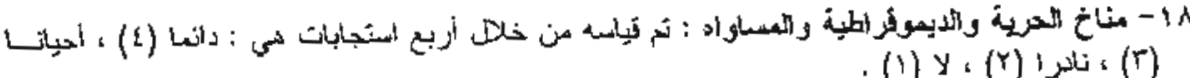

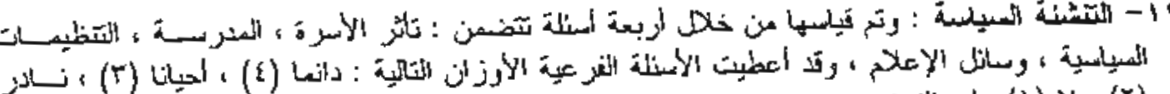

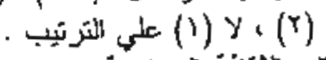

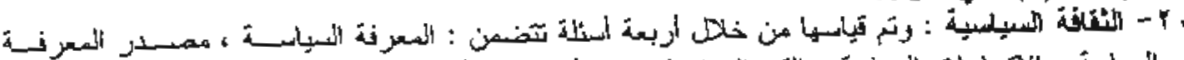

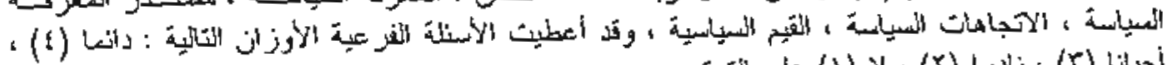

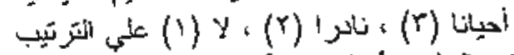

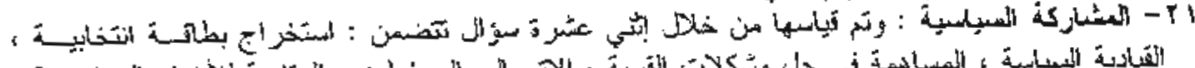

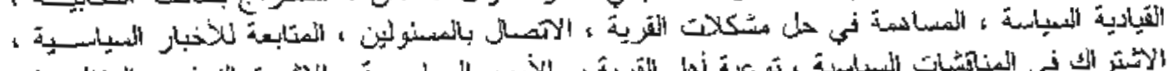

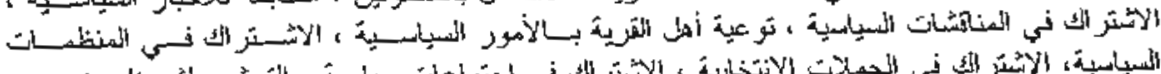

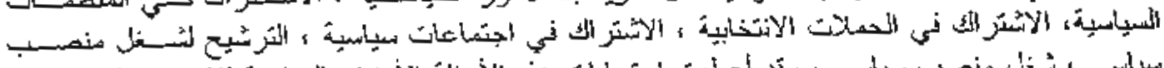

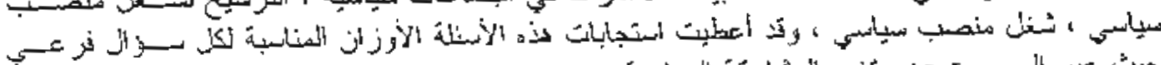

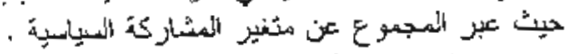

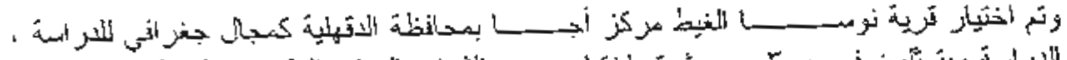

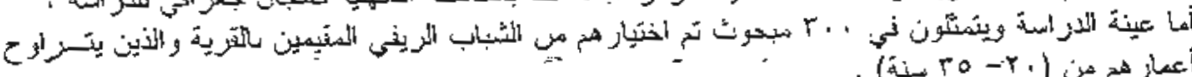

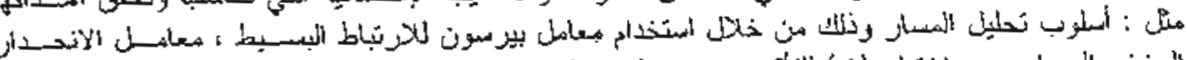

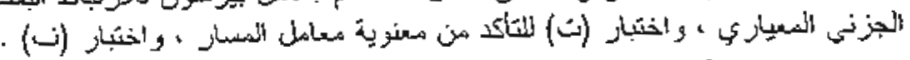

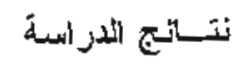

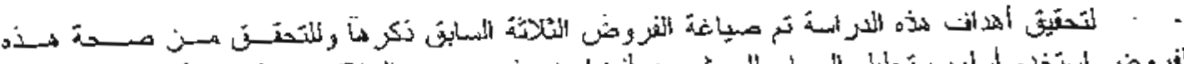

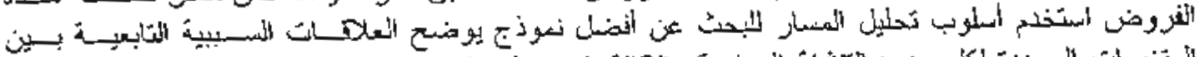

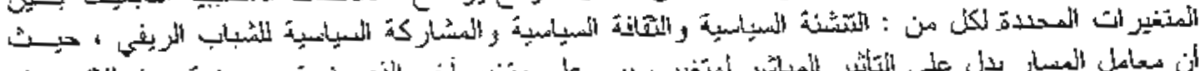

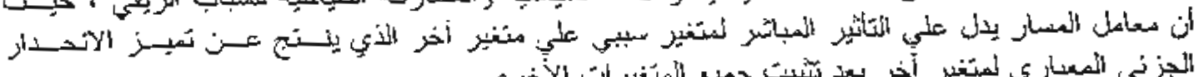

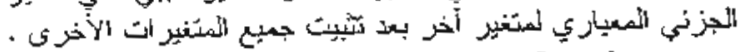

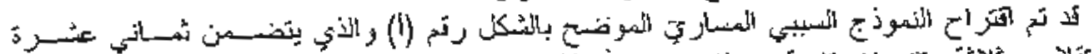

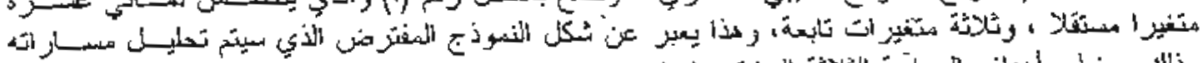

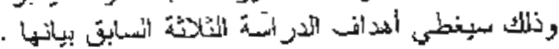


Abou- Hussein, Ibtehal M.K.

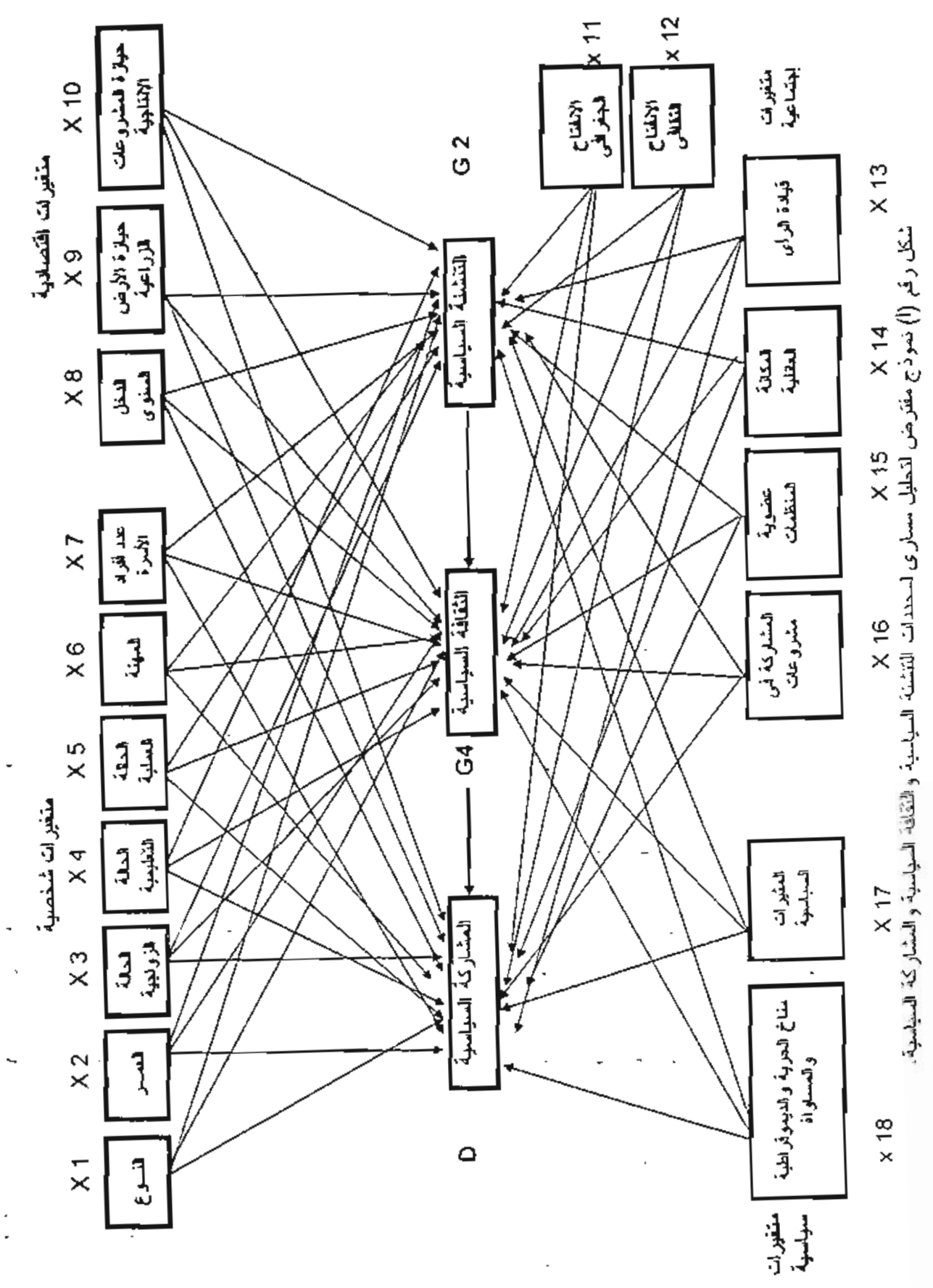




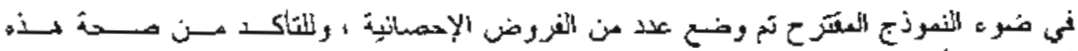

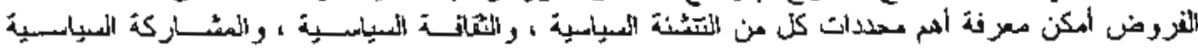

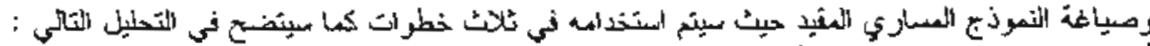

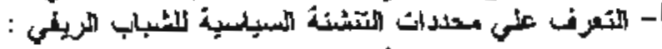

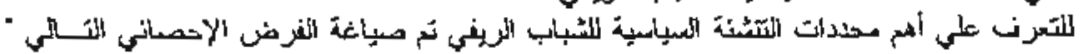

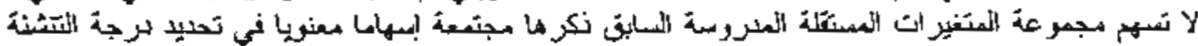

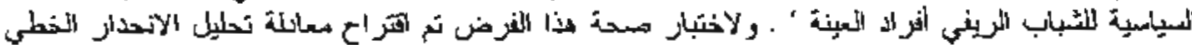

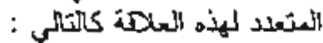

$\times 19=P 19 \times 1+P 19 \times 2+P 19 \times 3+P 19 \times 4+P 19 \times 5+P 19 \times 6+P 19 \times 7+P 19 \times 8$ $+P 19 \times 9+P 19 \times 10+P 19 \times 11+P 19 \times 12+P 19 \times 13+P 19 \times 14+P 19 \times 15+$ $P 19 \times 16+P 19 \times 17+P 19 \times 18+E 19$.

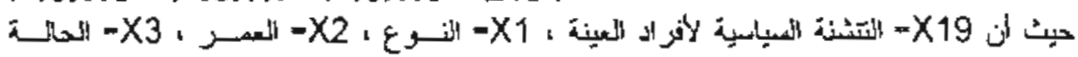

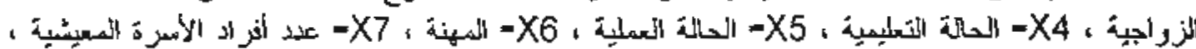

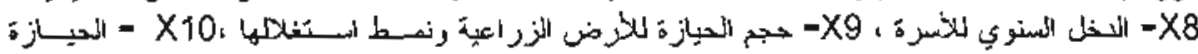

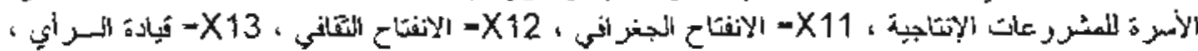

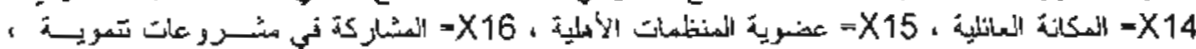

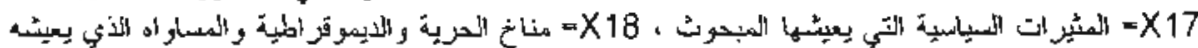

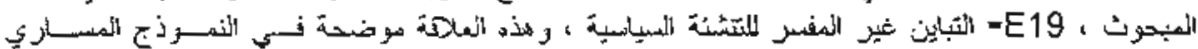

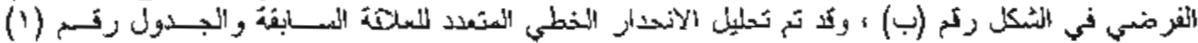

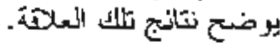

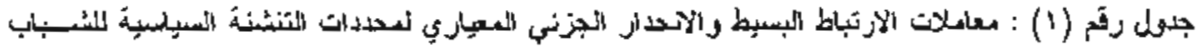

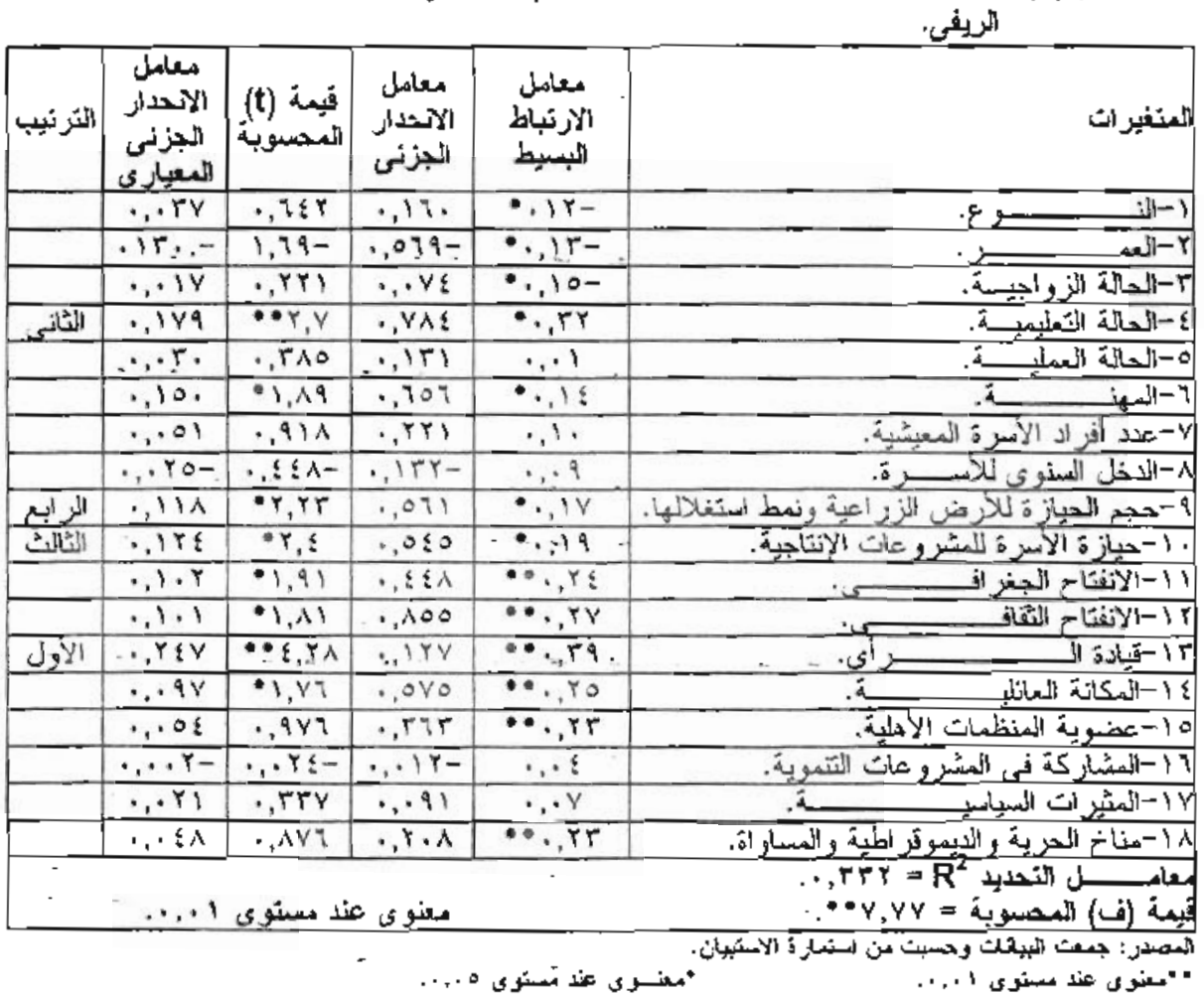


Abou- Hussein, Ibtehal M.K.
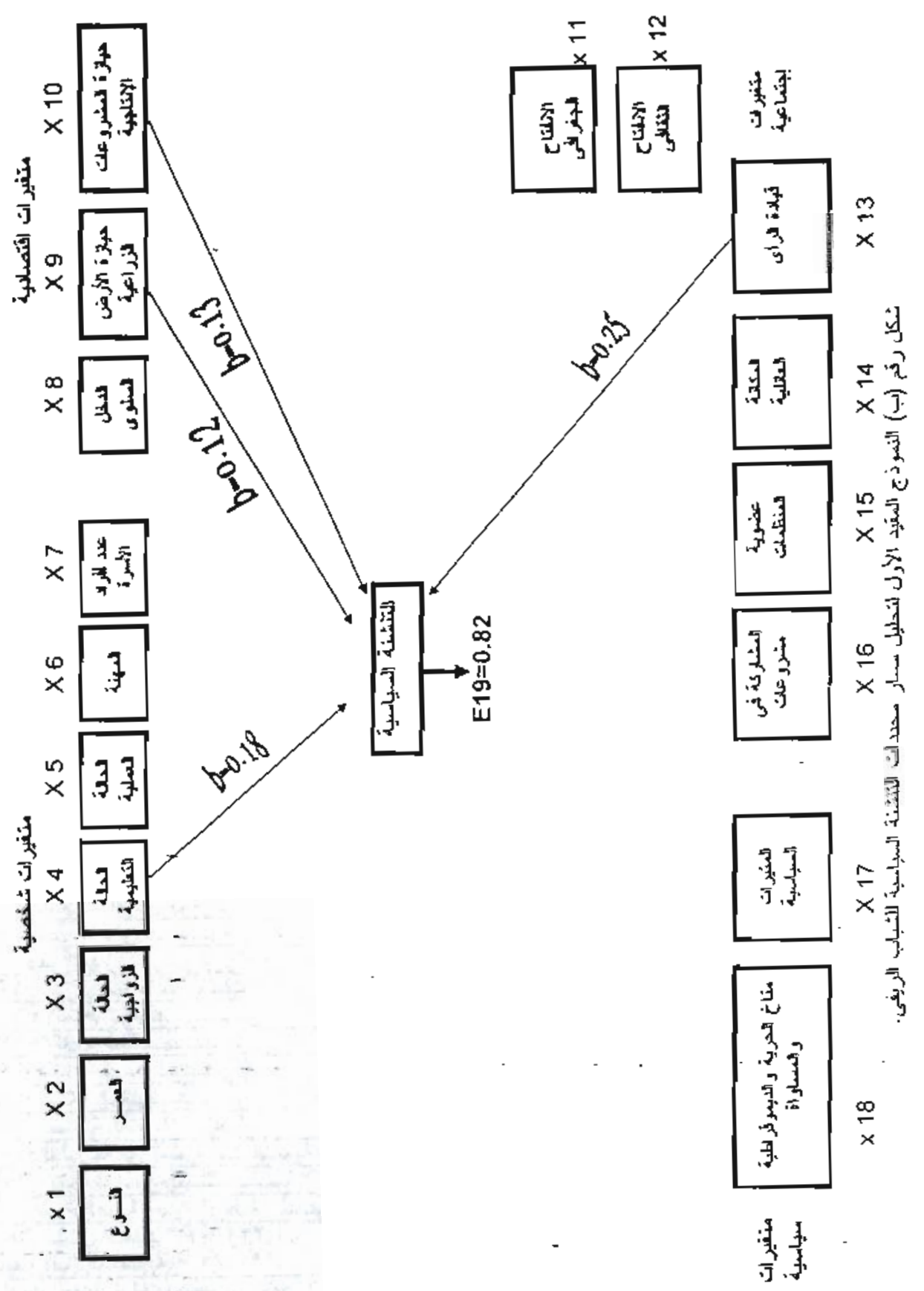


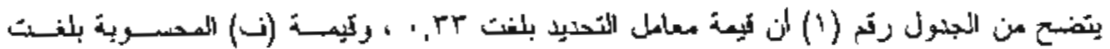

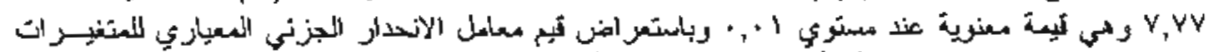

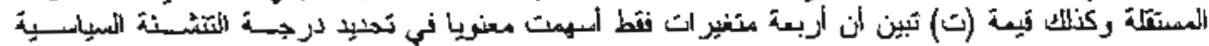

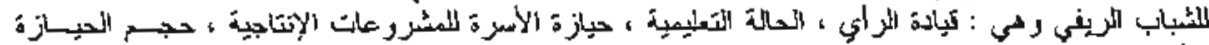

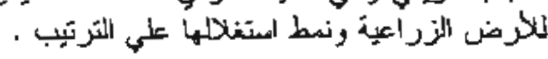

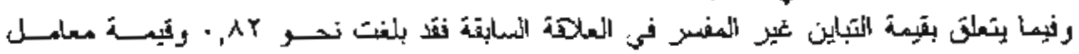

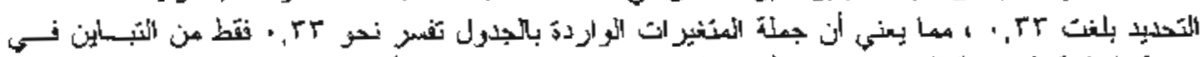

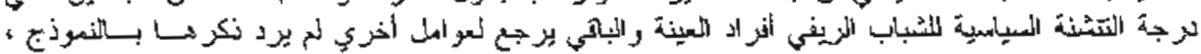

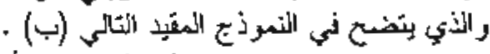

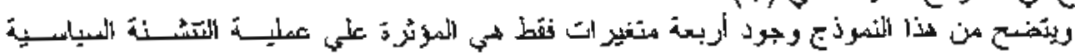

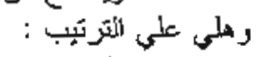

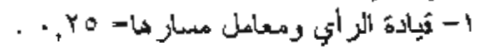

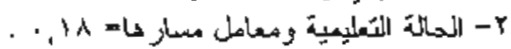

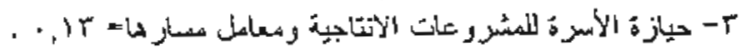

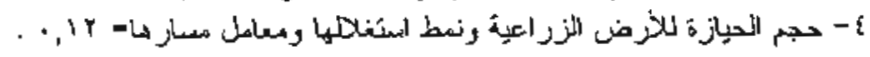

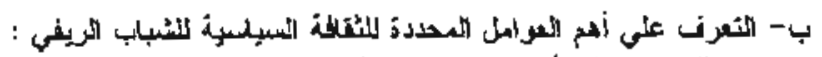

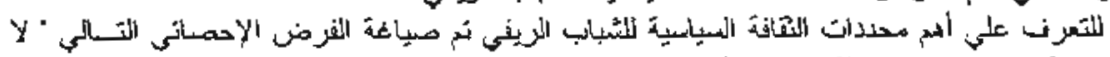

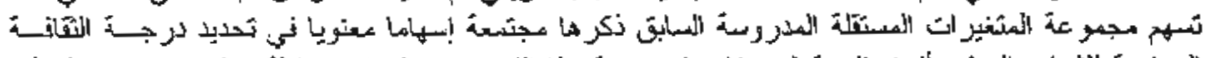

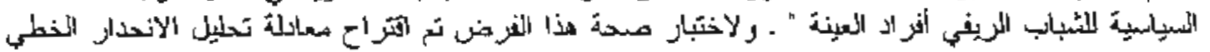

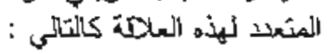

$\times 20=P 20 \times 1+P 20 \times 2+P 20 \times 3+P 20 \times 4+P 20 \times 5+P 20 \times 6+P 20 \times 7+P 20 \times 8$

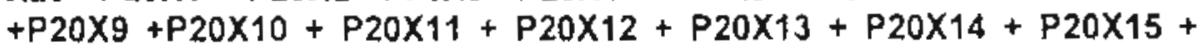
$P 20 \times 16+P 20 \times 17+P 20 \times 18+P 20 \times 19+E 20$.

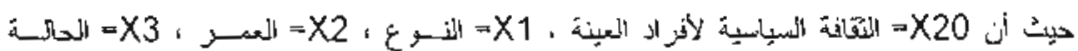

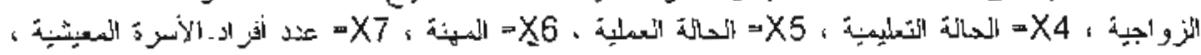

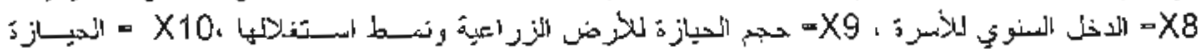

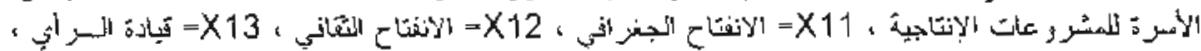

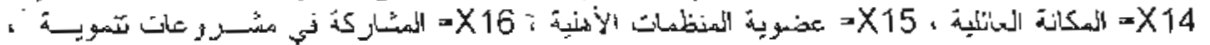

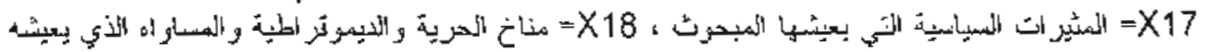

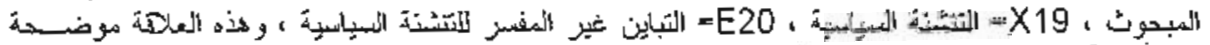

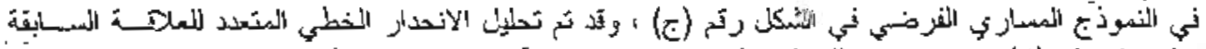

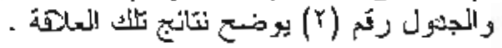


Abou- Hussein, Ibtehal M.K.

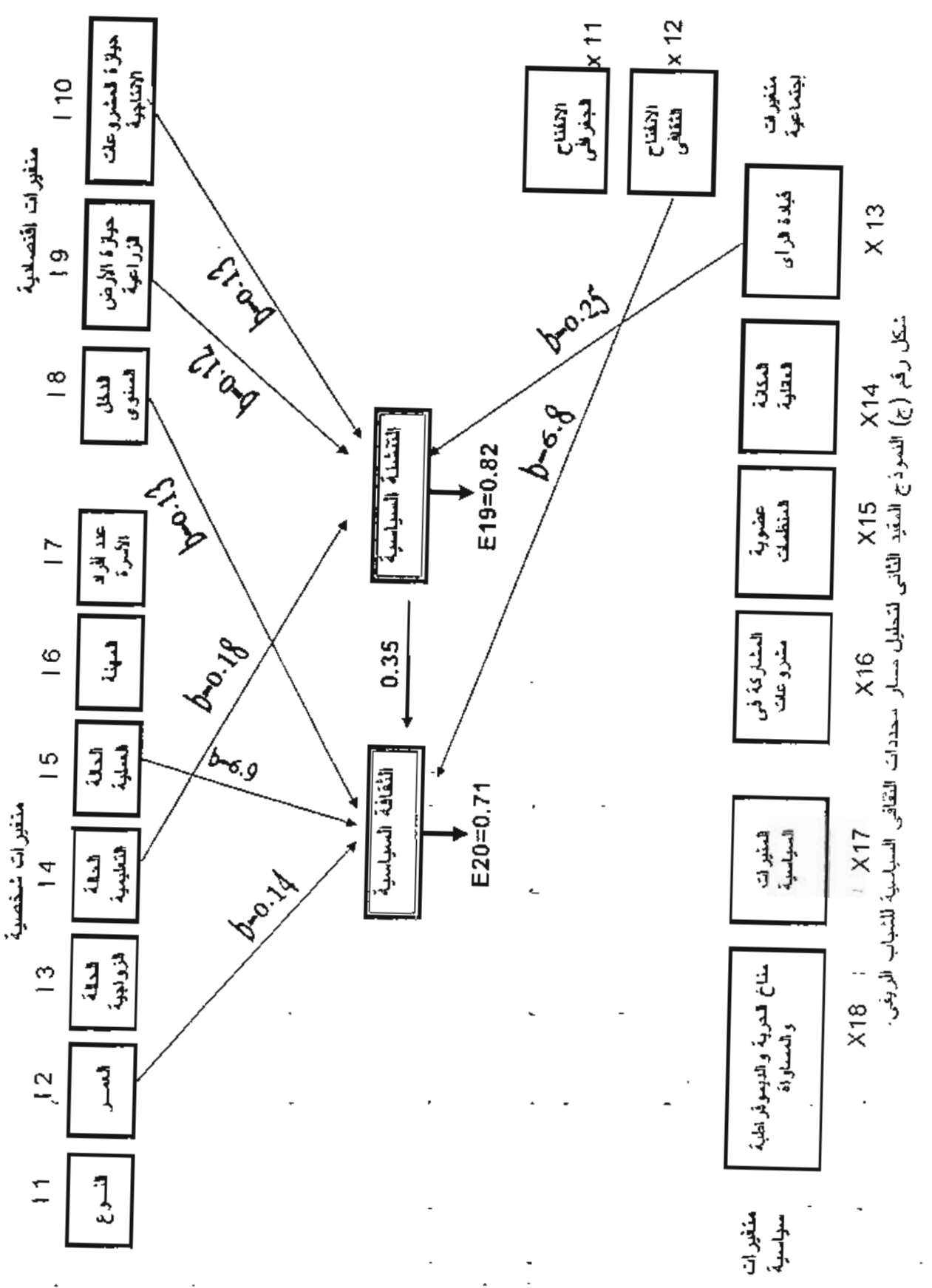


J. Agric. Sci. Mansoura Univ., 30 (4), Aprll, 2005

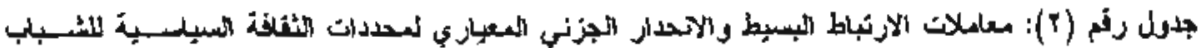

\begin{tabular}{|c|c|c|c|c|c|}
\hline النزتُبي & 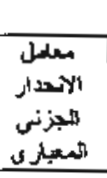 & 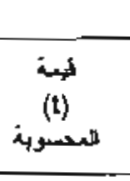 & 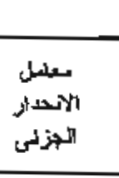 & 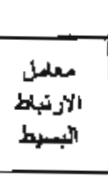 & ألرئئ. \\
\hline & $.4 \pi 4$ & 1,14 & $.10 \mathrm{~A}$ & $\cdots ., 19$ & $\varepsilon$ \\
\hline بالبز & $\therefore 1 T \mathrm{~A}$ & 4,98 & $.17 \mathrm{~A}$ & $\therefore, y-$ & 1- ד \\
\hline & 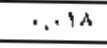 & $\cdot, Y \times Y$ &., $\mathrm{BY}$ & $\therefore, 1 \pm-$ & 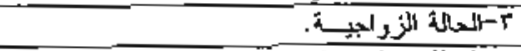 \\
\hline & $\cdot ., 92$ & 1.10 & $\sqrt{\cdot, 14}$ & $\because \because, 4$ & 侮 \\
\hline 1,4 & 911 & $\cdots 4,30$ &., $5 r_{0}$ & .7 & 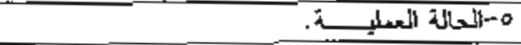 \\
\hline & $\because \cdot \mathrm{VT}$ & $\therefore$ AYh &.,$\overline{401}$ &., 8 & 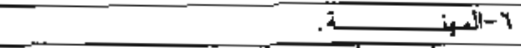 \\
\hline &. .919 & .919 & $\therefore n$ & 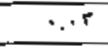 & 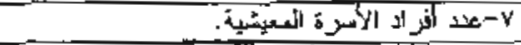 \\
\hline أن'اسن & $1,151-$ & $9.94-$ & $.1 \times 1-$ & 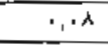 & ي - \\
\hline & $\because . Y_{9}$ & $.4 r$ &., $\mathrm{Vr} !$ & $\because 4$ & 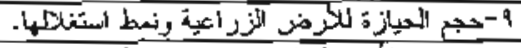 \\
\hline &., $.4 \pi$ &.,- YOY &., 179 & $\because \cdots$ & 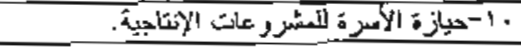 \\
\hline & $\therefore .10-$ & 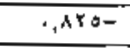 & $.90 n-$ & $\because 1.17$ & 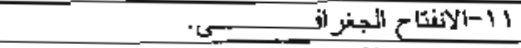 \\
\hline أثلانتي & $3, \lambda \Gamma-$ & $\cdots !, 19=$ &., $519-$ & $\because 1$ & - \\
\hline &.,$\times 1$ & $0.29 \mathrm{~V}$ & $-.51 \times$ & 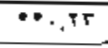 & 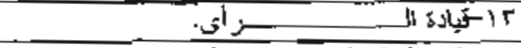 \\
\hline & $\because-34-$ &., $0 Y \leq=$ & $.00 \%-$ & $\because .1 T$ & 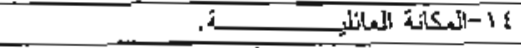 \\
\hline & $\because 1$ &., 19 & $\cdot 4.7$ & $\because \cdot, 18$ & 10 \\
\hline & $-.9-$ & $.917=$ &., $\mathrm{N}_{44-}$ & $\because .17$ & 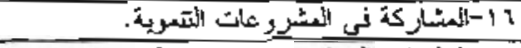 \\
\hline & $\therefore+4=$ & $.5,5-$ &., $\mathrm{rri-}$ & $\because, 4 r$ & 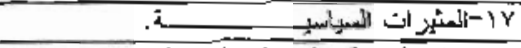 \\
\hline & $\ldots$ &.,$\cdots$ & $\cdot ., 144$ & $\because \cdot 9.10$ & 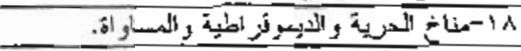 \\
\hline 15 & TOT & $\because 1, r !$ & $.5 \times 9$ & $\therefore \overline{\nabla T}$ & 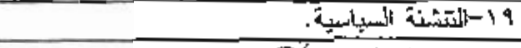 \\
\hline \multicolumn{5}{|c|}{ 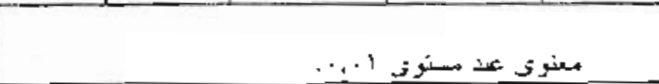 } & 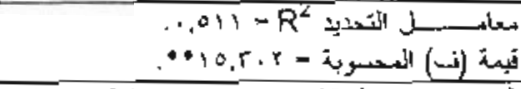 \\
\hline
\end{tabular}

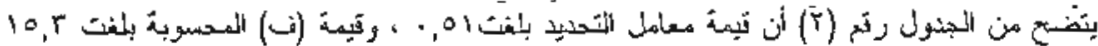

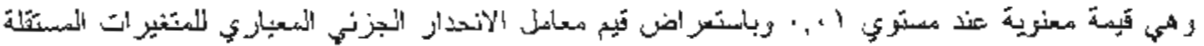

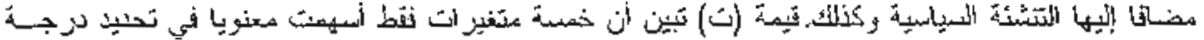

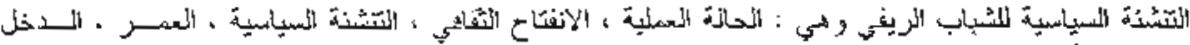

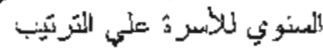

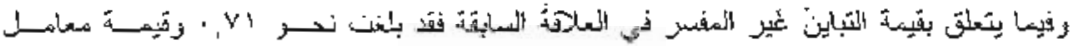

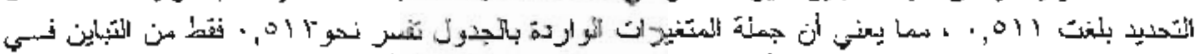

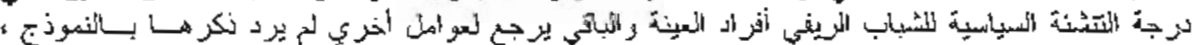

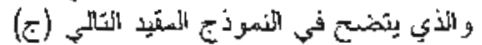

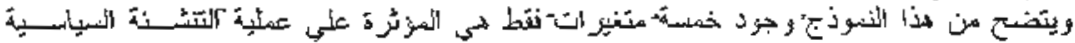

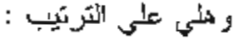

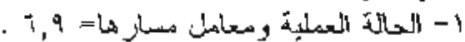

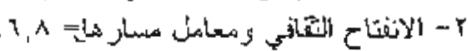

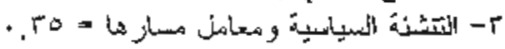

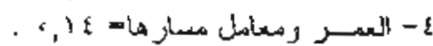

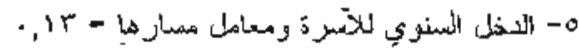




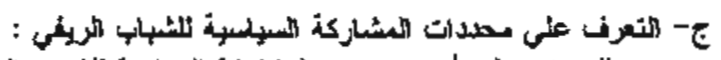

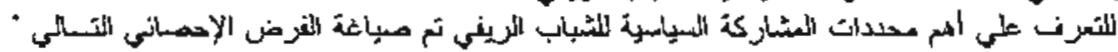

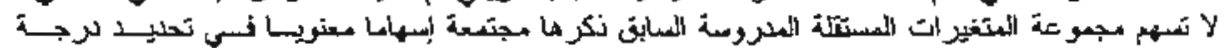

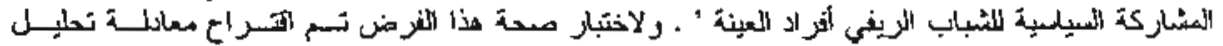

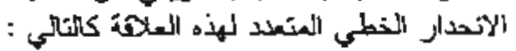
$\mathrm{X} 21=\mathrm{P} 21 \mathrm{X} 1+\mathrm{P} 21 \mathrm{X} 2+\mathrm{P} 21 \mathrm{X} 3+\mathrm{P} 21 \mathrm{X} 4+\mathrm{P} 21 \mathrm{X} 5+\mathrm{P} 21 \mathrm{X} 6+\mathrm{P} 21 \mathrm{X} 7+\mathrm{P} 21 \times 8$ $+\mathrm{P} 21 \times 9+\mathrm{P} 21 \times 10+\mathrm{P} 21 \times 11+\mathrm{P} 21 \times 12+\mathrm{P} 21 \times 13+\mathrm{P} 21 \times 14+\mathrm{P} 21 \mathrm{X} 15+$ $P 21 \times 16+P 21 X 17+P 21 \times 18+P 21 X 19+P 21 \times 20+E 21$.

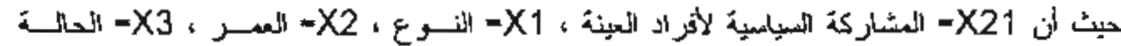

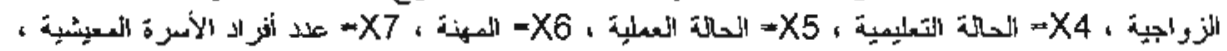

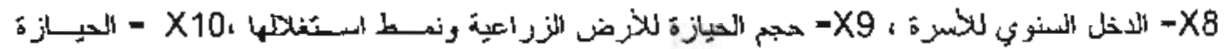

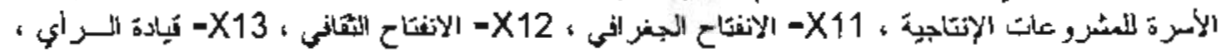

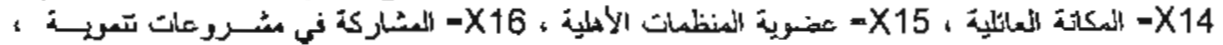

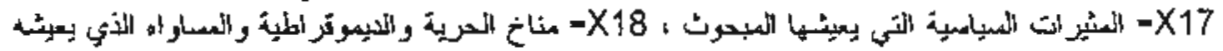

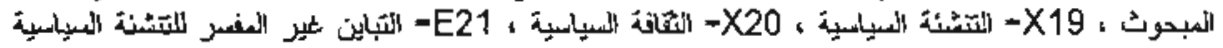

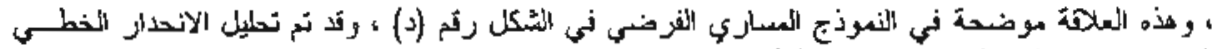

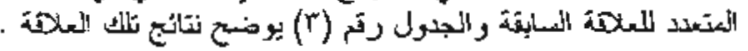

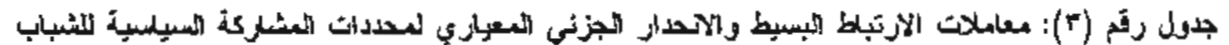

\begin{tabular}{|c|c|c|c|c|c|}
\hline الثرتب & 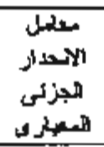 & العسئ & الاكتخلئل & "الارتبائل & الهتخيزتك \\
\hline انلربع & $.1 \% \mathrm{rt}$ & 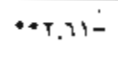 & $.011-$ & $\therefore$ & 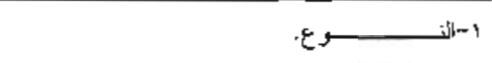 \\
\hline \multirow[t]{2}{*}{ آن } & 0,101 & 8,9 & ..004 & $\because \times 4 q$ & rا-rالعت \\
\hline & $\therefore .+\mathrm{ri}$ & $\cdot ., 499-$ & $\overline{0.479-}$ & $. .0 \%-$ & 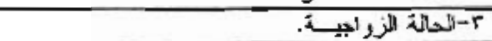 \\
\hline \multirow[t]{5}{*}{ 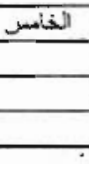 } &. $.1 \mathrm{rt}$ & $\cdots r_{1}, 9$ & 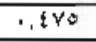 & $\cdots,{ }^{4}$, & 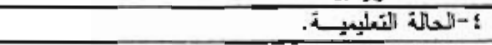 \\
\hline & $\therefore, \ldots$ & $\therefore .7 \mathrm{Y}-$ & $.9 \% \times-$ & $\because . \overline{\mathrm{Y} Y}-$ & 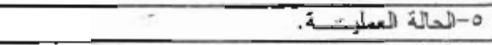 \\
\hline & $\because \because !-$ &., $1 \times 9-$ & $\therefore \frac{2}{2} m^{2}-$ & $\cdot, 1 \leq Y$ & $\therefore \quad 4+i-i$ \\
\hline & $\therefore ., \cdot$ &.,$\times 49$ & -.180 & 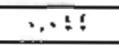 & 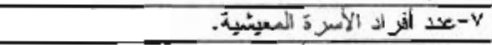 \\
\hline & $\therefore .0 \mathrm{~V}$ & $\frac{1.24}{4}$ &.,$r_{6}$ & $2 \cdot, 4,4$ & ي \\
\hline \multirow[t]{4}{*}{ ألسائمري } &., $17 t$ & $\cdot 9,4 !$ &., $\mathrm{BT}$ & $\cdot .1 \leqslant \mathrm{~A}$ & 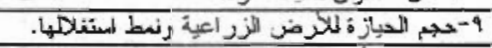 \\
\hline & $\because \cdot-\mathrm{T} A-^{-}$ &.,$\Delta \% D_{-}$ &., $1.5-$ & +.49 & 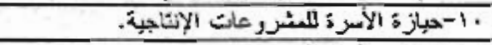 \\
\hline & $.1 \% \pi$ & $\cdot 9 ., 9$ &., 459 & $\because *,+4 a r$ & 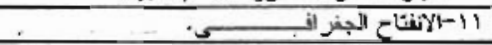 \\
\hline &. .94 & $=1, \mathrm{n}$ & 1.10 & $\because, \mathrm{YYT}$ & 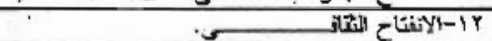 \\
\hline \multirow[t]{2}{*}{ Filin } &., 195 & 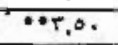 & $\cdot ., A 14$ & $* *, 6.0$ & 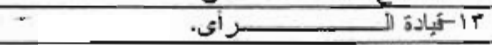 \\
\hline & $\therefore .79-$ & ,Yo!- &., 144 & $\cdot \cdot, 1: T$ & 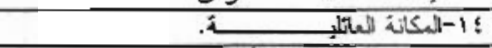 \\
\hline \multirow[t]{6}{*}{ Jyוn } & $\cdot . r \cdot 1$ & $\because 0,1 T$ & .1140 & $\because, t>t$ & 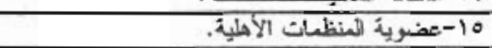 \\
\hline & $\because+4$ &.,$- T \leq 0$ & $\cdot, 11 \mathrm{~A}$ & $\therefore, 15:$ & 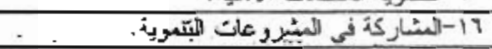 \\
\hline &.. $.1 \%$ & 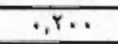 & $.8 \mathrm{str}$ & $\because, 110$ & با \\
\hline & $\therefore, \mathrm{ri}$ & 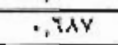 &., $1 T \mathrm{~A}$ & 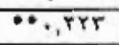 & 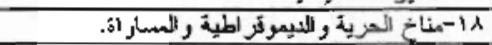 \\
\hline & $\therefore r$ &.. $\mathrm{TY}$ &., $1 \times 4$ &.,$\leqslant \mathrm{YA}$ & 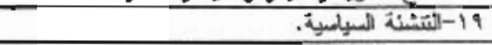 \\
\hline &., 1 &., 59 &. $.15 !$ &. .570 & . \\
\hline & & & - & & 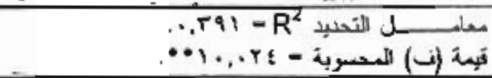 \\
\hline
\end{tabular}




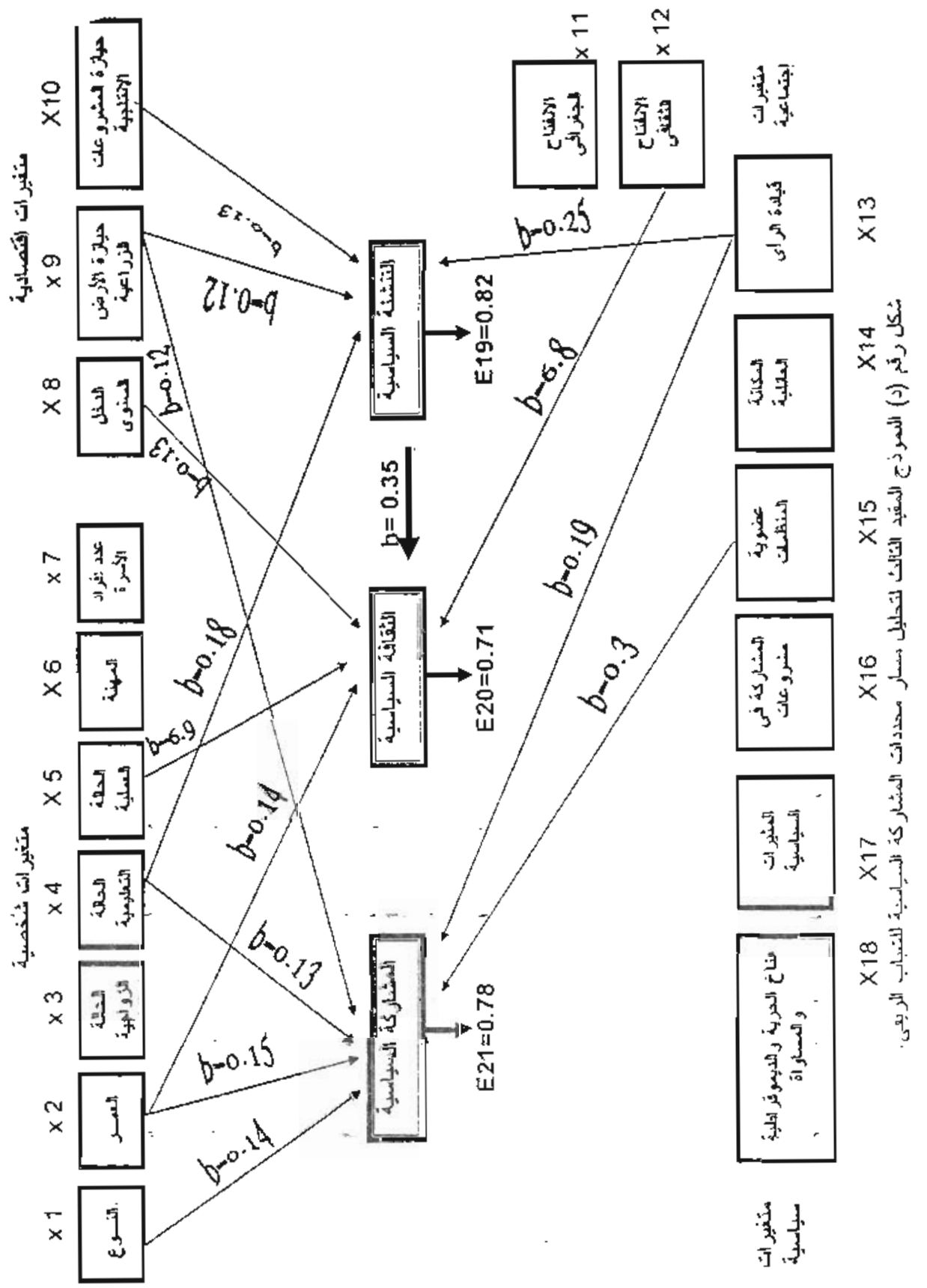




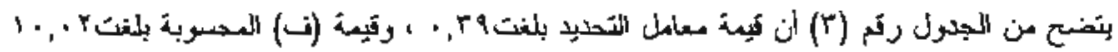

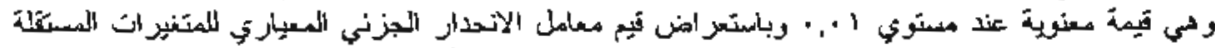

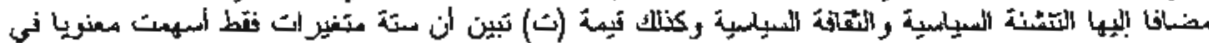

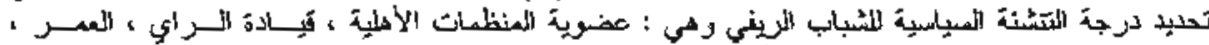

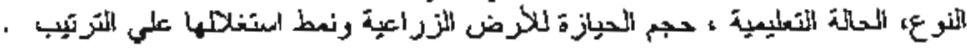

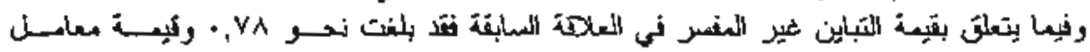

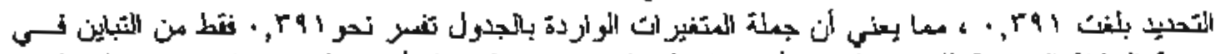

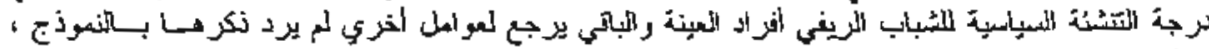

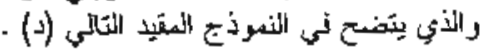

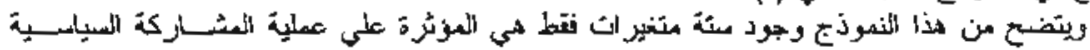

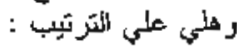
1

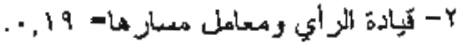

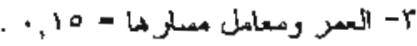

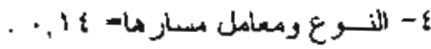

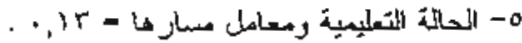

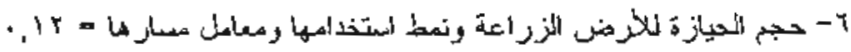

\section{مقتر حات البار المسية}

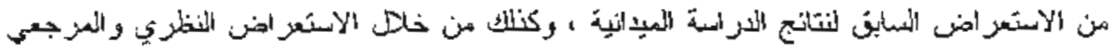

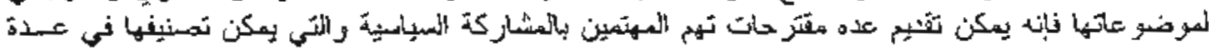

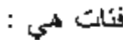

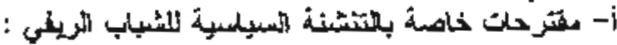

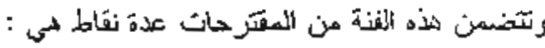

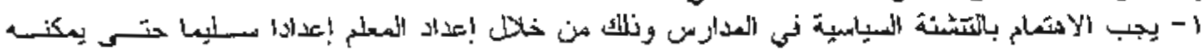

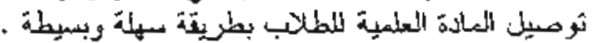

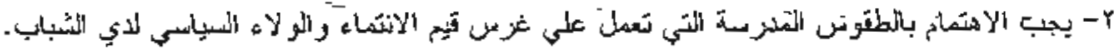

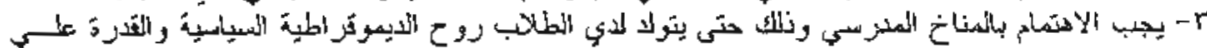

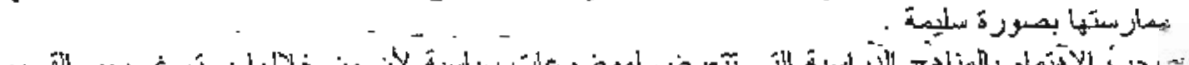

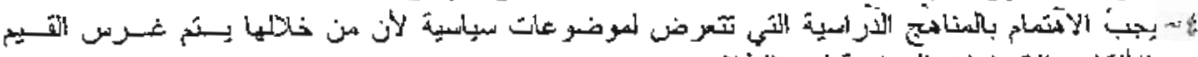

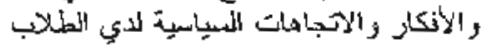

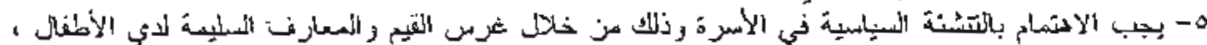

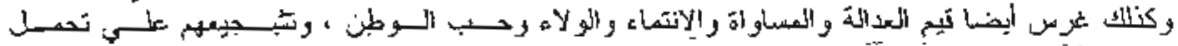

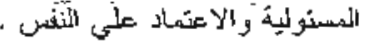

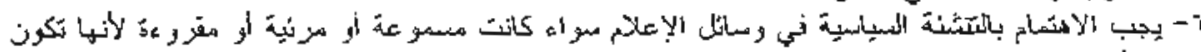

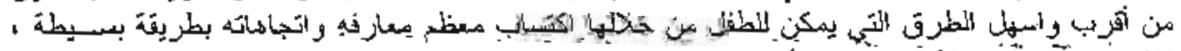

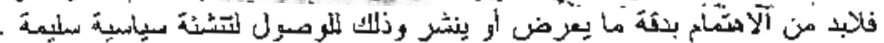

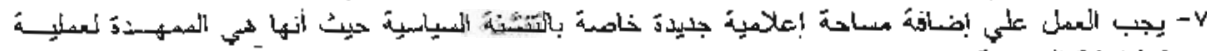

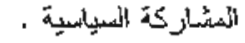

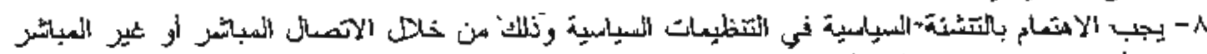

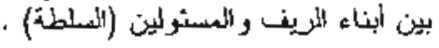




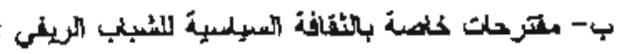

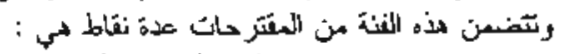

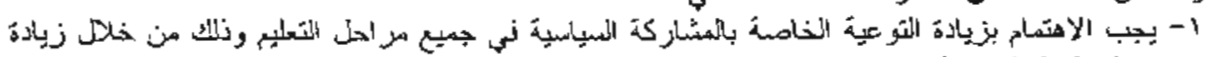

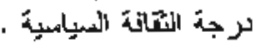

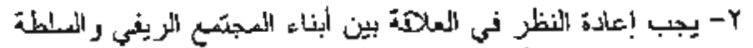

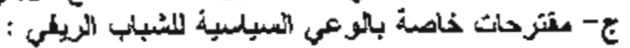

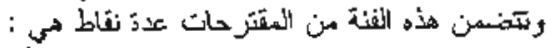

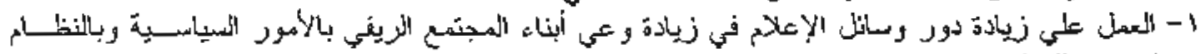

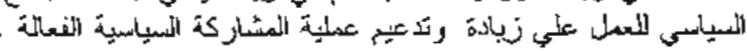

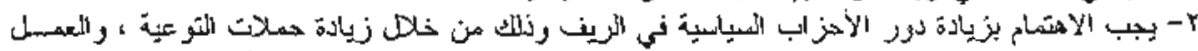

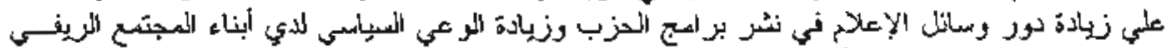

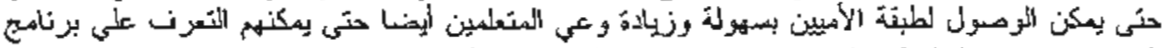

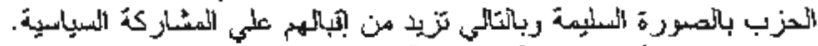

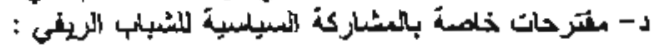

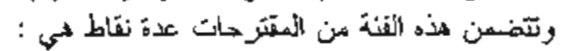

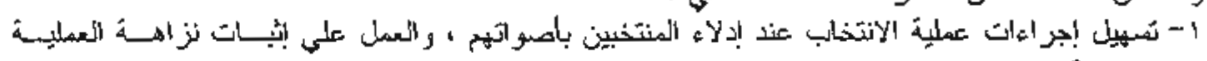

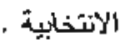

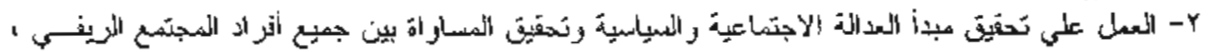

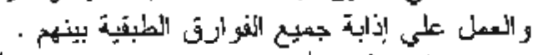

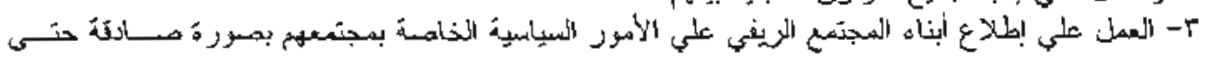

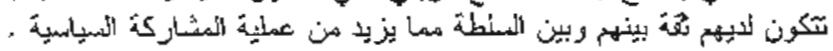

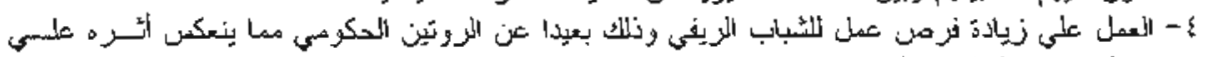

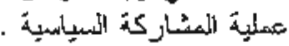

$$
\text { ألمراجي }
$$

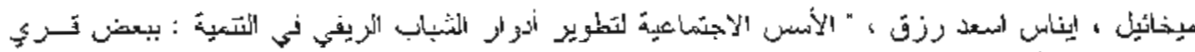

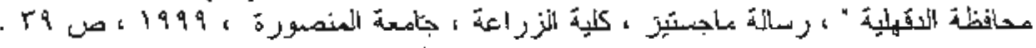

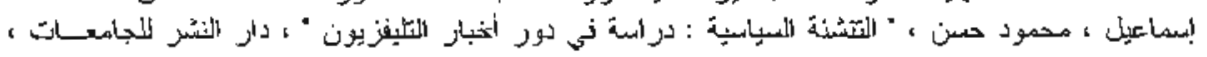

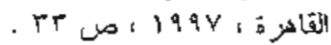

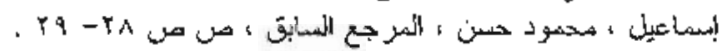

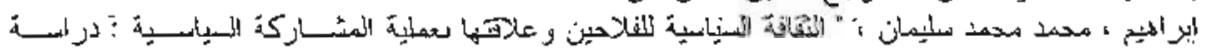

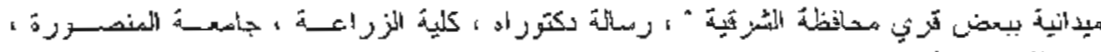

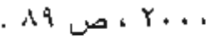

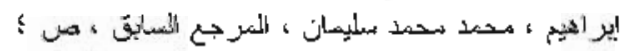

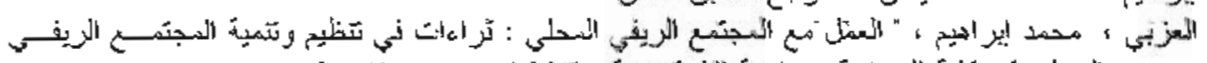

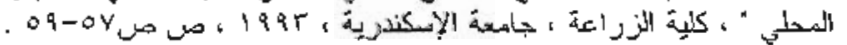

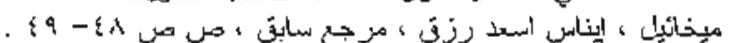

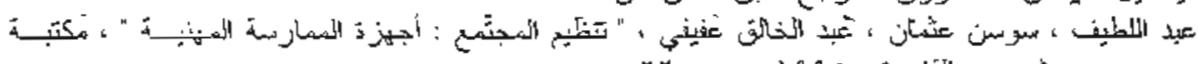

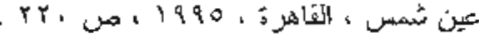

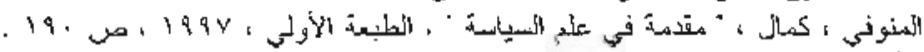

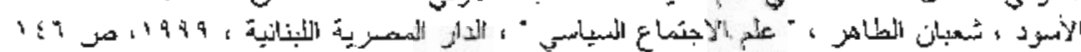

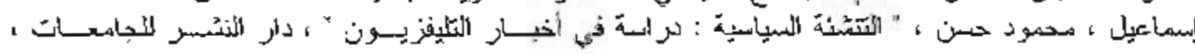

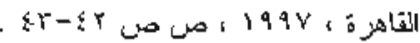

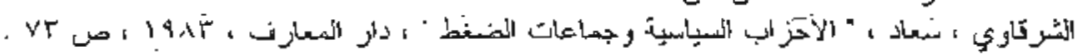




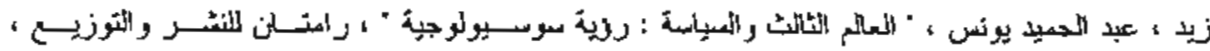
, TVA ט...

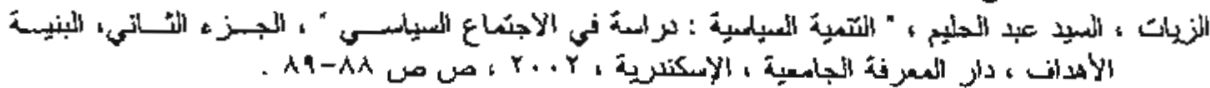

Sulaiman, Michel, "Socialization to politics in Moraco " international journal of middel east studies, 1985.

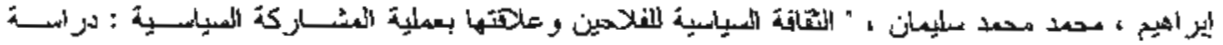

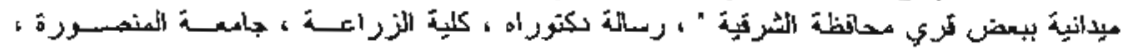

. Y...

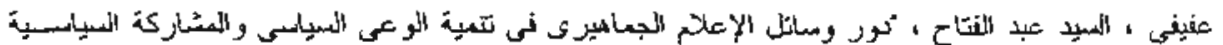

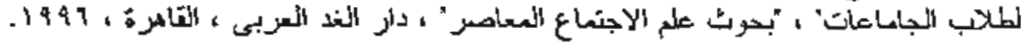

SOCIAL ANALYSIS FOR POLITICAL PARTICIPATION OF RURAL YOUTH: A FIELD STUDY ON RURAL YOUTH SAMPLE IN ONE VILLAGE AT DAKAHLIA GOVERNORATE Abou- Hussein, lbtehal M.K.

Agricultural Extension and Rural Soclology Department, Faculty of Agriculture Mansoura University .

\section{ABSTRACT}

This study aims : khowelage of determinants of the politicalization, the politica! culture, and the political participation of the rural youth .

This study applied in Nawasa El-Gheet village at Dakahlia Govemorate, and applied on random sample from rural youth (20-35 year). The size of sample $(300$ youth)

The researcher has used the frequencies,person correlation coefficient, partial linear regression, ascending multipie linear regression, path analysis-method, and (T) test in analyzing the data, and (f) test.

* Payh analysis of the determinants of the politicalization, the political culture . - and the political participation of the rural youth :

(1) The results of the first step of the analysis have shown that the most independent studied variables have contributed in specifying the politicalization degree of the rural youth are: the opinion leadersnip, the educational status and the occupation .

(2) The results of the second step of the analysis have shown that the most independent studied variables have contributed in specifying the political culture degree of the rural youth are : the practical status, the openness and politicalization

(3) The results of the third step of the analysis have shown that the most independent studied variables have contributed in specifying the political participation degree of the rural youth are : the membership in nongovernmental organization, the family position, and the age.

It has been shown that the politicalization significantly has contibuted in specifying the political culture of the rural youth of the samples. But the politicalization and the political culture of the samples slightly have in specifying the political participation degree. 